CONFORMAL GEOMETRY AND DYNAMICS

An Electronic Journal of the American Mathematical Society

Volume 9, Pages 76-101 (November 29, 2005)

S $1088-4173(05) 00124-4$

\title{
SPHERICALIZATION AND FLATTENING
}

\author{
ZOLTÁN M. BALOGH AND STEPHEN M. BUCKLEY
}

\begin{abstract}
The conformal deformations of flattening and sphericalization of length metric spaces are considered. These deformations are dual to each other if the space satisfies a simple quantitative connectivity property. Moreover, the quasihyperbolic metrics corresponding to the flat and the spherical metrics are bilipschitz equivalent if a weaker connectivity condition is satisfied.
\end{abstract}

\section{INTRODUCTION}

Recent progress in geometric function theory: $\mathrm{AG}$, AK], Se1, Se2, led to the study of conformal deformations of domains in Euclidean space. Elaborate results on quite general deformations defined on the unit ball have been developed in several recent papers, notably $[\mathrm{BKR}, \overline{\mathrm{BK}}, \mathrm{BHR}$. The extension of the theory of quasiconformal mappings from Euclidean to general metric spaces [HK], $\mathrm{BKO}$, Ty1, Ty2 motivated the study of conformal deformations in general metric spaces $\mathrm{BHK}, \mathrm{BB}, \mathrm{He} 1, \mathrm{He} 2$.

One of the most important conformal deformations is the so-called quasihyperbolic metric $k$ which, on a domain $\Omega \subsetneq \mathbb{R}^{n}$, is defined by the equation

$$
k_{\Omega}(x, y)=\inf _{\gamma \in \Gamma(x, y)} \int_{\gamma} \frac{d s(z)}{\operatorname{dist}(x, \partial \Omega)},
$$

where $\Gamma(x, y)$ is the class of rectifiable paths $\lambda:[0, T] \rightarrow X$ for which $\lambda(0)=x$ and $\lambda(T)=y, d s$ is the Euclidean length element, and $\operatorname{dist}(x, \partial \Omega)$ is the Euclidean distance to the boundary. This metric was extensively studied in $\mathrm{BHK}, \mathrm{He} 2$, $\mathrm{BB}$. Notice however that if we instead use the spherical length element and spherical boundary distance, we get an alternate quasihyperbolic metric $k_{\Omega}^{\prime}$. Both $k_{\Omega}$ and $k_{\Omega}^{\prime}$ are used frequently in the literature, but how do they compare? This question arises naturally from the results of $[\mathrm{BHK}]$ and $[\mathrm{BB}]$ which were proven for the spherical quasihyperbolic metric $k_{\Omega}^{\prime}$; but in many instances one would like to know what happens for the case of the usual quasihyperbolic metric $k_{\Omega}$.

Notice first that $k_{\Omega}(x, y)$ can be much smaller than $k_{\Omega}^{\prime}(x, y)$. For instance, if $\Omega$ is a ball of Euclidean radius $r>2$ centered at the origin, $x$ is the origin, and $y$ is a point on the Euclidean unit sphere then, as $r \rightarrow \infty, k_{\Omega}(x, y)$ tends to 0 but $k_{\Omega}^{\prime}(x, y)$ is bounded away from 0 . Despite this example, we will prove the following result in $\S 2.24$.

Received by the editors October 26, 2004 and, in revised form, September 28, 2005.

2000 Mathematics Subject Classification. Primary 30F45.

This research was partially supported by the Swiss Nationalfond and Enterprise Ireland. It was partly conducted during a visit by the second author to the University of Bern; the hospitality of the Mathematics Department was much appreciated.

(C)2005 American Mathematical Society Reverts to public domain 28 years from publication 
Theorem 0.1. Given $x_{0} \in \mathbb{R}^{n}$ and $r_{0}>0$, let $\mathcal{C}$ be the class of all domains $\Omega \subsetneq \mathbb{R}^{n}$ containing $x_{0}$ for which $\operatorname{dist}\left(x_{0}, \partial \Omega\right) \leq r_{0}$. For $\Omega \in \mathcal{C}$ the quasihyperbolic metrics $k_{\Omega}, k_{\Omega}^{\prime}$ defined above are bilipschitz equivalent to each other, with a bilipschitz constant dependent only on $\left|x_{0}\right|$ and $r_{0}$.

In order to obtain this statement we have to consider carefully the relation between the spherical and the Euclidean metric. In fact we examine the relation between two classes of conformal deformations in a general metric space setting. We call the first class of deformations sphericalization. If we sphericalize an unbounded length space, we get an bounded length space which is usually incomplete; an example is the process of deforming Euclidean space into the Riemann sphere minus the north pole. We call the second class of deformations flattening. If we flatten an incomplete bounded length space, we get an unbounded length space; an example is the process of deforming the Riemann sphere minus the north pole into Euclidean space. In the process of flattening, a selected part of the boundary (such as the north pole) is pushed out to infinity, while sphericalization brings the boundary at infinity back to a bounded distance. These two procedures are dual to each other in a similar way as the procedures of quasihyperbolization and dampening from $\mathrm{BHK}$. We show in a general metric setting that a necessary and sufficient condition for the quasihyperbolic metric associated with the original metric to be bilipschitz equivalent to the quasihyperbolic metric associated with the sphericalized metric (or flattened metric) is a surprisingly simple geometric property of the original space which we call an escape property (or reverse escape property). Since all Euclidean domains have such properties, Theorem 0.1 will follow almost immediately.

As already indicated, the main motivation for this investigation lies in the results of $[\mathrm{BHK}$ and $\mathrm{BB}$. Those papers investigate the question of when the quasihyperbolic metric associated with a general length metric is Gromov hyperbolic. The assumption of boundedness is essential for some of the key results in those papers. Since one is often interested in quasihyperbolic metrics on unbounded Euclidean domains and other unbounded metric spaces, this analysis at first seems to be of limited use in that context. However on a large class of unbounded spaces (including all Euclidean domains), the quasihyperbolic metric on the original (unbounded) space is Gromov hyperbolic if and only if the quasihyperbolic metric on the sphericalized (bounded) space is Gromov hyperbolic, allowing us in theory to apply the results of those previous papers to unbounded spaces. Since sphericalization is a fairly simple conformal distortion, it is often easy to get a rather explicit description of the sphericalized space in terms of the original space, so this method of appealing to the results of $[\mathrm{BHK}]$ and $\mathrm{BB}$ is in practice quite feasible.

After defining our main notation in Section 1, we introduce the class of conformal deformations called sphericalization and flattening and discuss the procedures of sphericalization in Section 2, and flattening in Section 3. We obtain, in conclusion, the comparability of the spherical and the flat quasihyperbolic metrics. In Section 4, we examine to what extent sphericalization and flattening are inverse processes. We introduce the notion of dual deformations in the context of sphericalizationflattening. We show that if we flatten a sphericalized space (or sphericalize a flattened space) using "dual" sphericalizing and flattening functions, then we get a space bilipschitz equivalent to the original one if and only if the original space satisfies a stronger variant of the escape property (or reverse escape property). This 
makes it easy to give examples of incomplete bounded spaces that are not produced by sphericalization, and unbounded spaces that are not produced by flattening.

\section{Preliminaries}

We write $A \lesssim B$ or $B \gtrsim A$ whenever $A \leq C B$ for some positive constant $C$ that depends only on explicitly allowed parameters, and we write $A \approx B$ if $A \lesssim B \lesssim A$. We denote by $a \vee b$ and $a \wedge b$ the maximum and minimum, respectively, of any $a, b \in[-\infty, \infty]$.

In later sections, $d, l$, and $\sigma$ will denote metrics on a space $X$ that are related to each other in special ways. In this section, we define some concepts that we need for one or more of these metrics, so we do so for a generic metric space $(X, m)$ (satisfying any additional conditions that are listed).

Given $x \in X$ and $r \in \mathbb{R}$, we define $B_{m}(x, r)=\{y \in X: m(y, x)<r\}$. We denote by $\bar{X}_{m}$ the metric completion of $X$ and $\partial X_{m}=\bar{X}_{m} \backslash X$. We say that $(X, m)$ is locally complete if $\operatorname{dist}_{m}\left(x, \partial X_{m}\right)>0$ for all $x \in X$; of course $(X, m)$ is complete if and only if $\operatorname{dist}_{m}\left(\cdot, \partial X_{m}\right) \equiv \infty$.

Given $x, y \in \bar{X}_{m}, \Gamma(x, y ; m)$ denotes the class of $m$-rectifiable paths $\gamma:[0, T] \rightarrow$ $\bar{X}_{m}$ for which $\left.\gamma\right|_{(0, T)}$ is a rectifiable path in $X, \gamma(0)=x$, and $\gamma(T)=y$. We also define $\Gamma_{m}(x, y)$ to be the subset of $\Gamma(x, y ; m)$ consisting of paths that are parametrized by $m$-arclength. If $\gamma$ is a path in $(X, m)$, then $\gamma(T-; m)$ is shorthand for $\lim _{t \rightarrow T-} \gamma(t)$, assuming this $m$-limit exists in $\bar{X}_{m}$, and $\operatorname{len}_{m}(\gamma)$ is the $m$-length of $\gamma$. We do not distinguish notationally between paths and their images.

1.1. Local length spaces. For $x, y \in \bar{X}_{m}$, let $l(x, y)=\inf _{\gamma \in \Gamma_{d}(x, y)} \operatorname{len}_{m}(\gamma)$. If $l l_{X \times X}$ is always finite, we call $l$ the inner metric associated with $m$, and say that $(X, m)$ is rectifiably connected. Recall that $(X, m)$ is a length space if $l(x, y)=$ $m(x, y)$ for all $x, y \in X$; such an equality clearly extends to points $x, y \in \bar{X}_{m}$. More generally, $(X, m)$ is a local length space, and $m$ is a local length metric, if $m$ is rectifiably connected and satisfies the following pair of conditions:

(a) If $x \in X, y \in \bar{X}_{m}$, and $m(x, y) \leq \operatorname{dist}_{m}\left(x, \partial X_{m}\right)$, then there is a path $\gamma \in \Gamma(x, y ; m)$ of arclength arbitrarily close to $m(x, y)$.

(b) If $x \in X$, then $\operatorname{dist}_{l}\left(x, \partial X_{l}\right)=\operatorname{dist}_{m}\left(x, \partial X_{m}\right)$.

Note that a complete local length space is trivially a length space. Also note that condition (b) is necessary to ensure the existence of short paths to the boundary in the case where the infimum $\inf _{z \in \partial X_{m}} m(x, z)$ is not attained.

In this paper, we are particularly interested in locally complete local length spaces. Examples of such spaces include all Euclidean domains, and more generally, all rectifiably connected open subsets of complete length spaces. Note that most examples of this type are neither complete nor length spaces (consider, for instance, the slit disk in $\mathbb{R}^{2}$ ).

1.2. Metric and accessible boundary. Let us pause to make some basic comments on the relationship between $\bar{X}_{m}$ and $\bar{X}_{l}$ when $(X, m)$ is a locally complete local length space, with associated inner metric $l$. Cauchy sequences in $(X, l)$ are also Cauchy in $(X, m)$, so there is a natural map $I: \partial X_{l} \rightarrow \partial X_{m}$. The rectifiably accessible boundary of $(X, m), \partial_{\mathrm{ra}} X_{m}:=I\left(\partial X_{l}\right)$, is the set of all $y \in \partial X_{m}$ whose inner distance from some (and hence all) $x \in X$ is finite. Equivalently,

$$
\partial_{\mathrm{ra}} X_{m}=\left\{y \in \partial X_{m}: \Gamma(x, y ; m) \neq \emptyset, x \in X\right\} .
$$


The map $I$ identifies each $y \in \partial_{\mathrm{ra}} X_{m}$ with a subset $I^{-1}(y)$ of $\partial X_{l}$.

If $m$ is a length metric, then trivially $\partial X_{m}=\partial_{\mathrm{ra}} X_{m}=\partial X_{l}$, but in other cases, there may be no natural identifications between these sets. In particular, the map $I: \partial X_{l} \rightarrow \partial X_{m}$ may be neither injective nor surjective. For instance, if $m$ is the Euclidean metric on a domain $\Omega \subset \mathbb{R}^{2}$ consisting of the complement of a sufficiently tight spiral about $y \in \mathbb{R}^{2}$, then $y$ corresponds to a point in $\partial \Omega_{m} \backslash \partial_{\mathrm{ra}} \Omega_{m}$, while if $\Omega \subset \mathbb{R}^{2}$ is the complement of a line segment $[a, b]$, then each point in the open line segment $(a, b)$ corresponds to two elements of $\partial \Omega_{l}$, but only to one point in $\partial_{\mathrm{ra}} \Omega_{m}$. Condition (a) above says that $\partial_{\mathrm{ra}} X_{m}$ contains at least all points $y \in \partial X_{m}$ for which there is an associated point $z=z_{y} \in X$ with $m(z, y)=\operatorname{dist}_{m}\left(z, \partial \Omega_{m}\right)$.

1.3. Metrics of quasihyperbolic type. Suppose $(X, m, o)$ is a locally complete rectifiably connected pointed metric space. Writing $\delta_{m, C}(x)=\operatorname{dist}_{m}\left(x, \partial X_{m}\right) \wedge$ $(C+m(x, o))$ for $0<C \leq \operatorname{dist}\left(o, \partial X_{m}\right)$, we define the $C$-quasihyperbolic metric on $X$ by the equation

$$
k_{m, C}(x, y)=\inf _{\gamma \in \Gamma(x, y ; m)} \int_{\gamma} \frac{d m(z)}{\delta_{m, C}(z)} .
$$

We allow the maximal choice $C=\operatorname{dist}\left(o, \partial X_{m}\right)$ only when $(X, m)$ is incomplete. Note that local completeness is essential to ensure that the right-hand side of (1.2) is finite. Whenever we omit the subscript $C$ in the above notation, it is to be assumed that $C$ is maximal (and $X$ is incomplete). Thus $\delta_{m}=\operatorname{dist}_{m}\left(\cdot, \partial X_{m}\right)$, and $k_{m}$ is the quasihyperbolic metric as it is usually defined for incomplete spaces, but nonmaximal choices of $C$ give variants of these concepts that are also defined on complete spaces.

In this paper, we compare quasihyperbolic metrics of the above type for two underlying metrics $m$, where one of the underlying metrics is obtained from the other one by either sphericalization or flattening. We will see that the comparison results do not depend on whether the above parameter $C$ is maximal or not.

\section{SPhericalization}

Throughout this section, $(X, d, o)$ is an unbounded locally complete pointed local length space, and we write $|x|=d(x, o), x \in \bar{X}_{d}$. We will "sphericalize" $(X, d, o)$ to get a related length space $(X, \sigma)$; we use the term "sphericalization" because this process generalizes that of obtaining the Riemann sphere from Euclidean space. The main result, Theorem 2.28, compares the corresponding quasihyperbolic metrics $k_{d, C}$ and $k_{\sigma}$. For a basic example of this process, let $(X, d)$ be a Euclidean domain. Then $\sigma$ is the inner spherical metric on $X$ (if we make the standard choice of sphericalizing function $g$ ). Alternatively, we can obtain the spherical metric by sphericalizing the whole of Euclidean space and restricting the resulting metric $\sigma$ to $\Omega$.

We denote by $l$ the inner metric associated with $d$. We will use $l$ only in two contexts: the length element $d l$ associated with $d$, and the $l$-boundary $\partial X_{l}$. We write $|x|=d(I(x), o)$ for all $x \in \partial X_{l}$ where $I: \partial X_{l} \rightarrow \partial X_{d}$ is the natural injection as in $\S 1.2$.

Given $g:[0, \infty) \rightarrow(0, \infty)$, we define a length metric $\mathfrak{S}(d, o, g)$ on $X$ by the equation

$$
\mathfrak{S}(d, o, g)(x, y)=\inf _{\gamma \in \Gamma(x, y ; d)} \int_{\gamma} g(|z|) d l(z), \quad x, y \in X .
$$


We normally write $\sigma$ in place of $\mathfrak{S}(d, o, g)$, and we take $g$ to be a sphericalizing function, a concept we now define.

2.1. Sphericalizing functions. A continuous function $g:[0, \infty) \rightarrow(0, \infty)$ is a $C$-sphericalizing function, $C>2$, if it has the following properties:

(S1) $g(r) \leq C g(s)$ whenever $r, s>0, r \leq 2 s+1$, and $s \leq 2 r+1$.

(S2) $\int_{r}^{\infty} g(t) d t \leq C r g(r), r \geq 1$.

We will see that this pair of properties is quantitatively equivalent to the combination of (S1) and the following condition, where $0<\epsilon \leq 1 \leq C^{\prime}$ are some other constants:

(S3) $\frac{g(s)}{g(r)} \leq C^{\prime}\left(\frac{r}{s}\right)^{1+\epsilon}$, for all $1 \leq r \leq s$.

Notice that (S1) merely says that all values of $g$ on any one of the intervals $I_{j}$ are mutually comparable, where $I_{0}=[0,1]$ and $I_{j}=\left[2^{j-1}, 2^{j}\right], j \in \mathbb{N}$.

Typical sphericalizing functions include the "standard sphericalizing function" $g(t)=2 /\left(1+t^{2}\right)$ and, more generally, $g(t)=c \log ^{q}(2+t) /\left(1+t^{p}\right)$, where $p>1$, $q \in \mathbb{R}$, and $c>0$. Note that (S1) and (S3) imply that $g$ is quasidecreasing in the sense that

(S4) $g(s) \leq C^{\prime \prime} g(r), 0 \leq r \leq s$, where $C^{\prime \prime}=C C^{\prime}$. Moreover $h(r):=\sup _{r \geq s} g(s)$ defines a monotonically decreasing $C_{1}$-sphericalizing function comparable to $g$, where $C_{1}=C^{2} C^{\prime}$. Thus we may assume without loss of generality that a sphericalizing function is decreasing whenever switching to a comparable sphericalizing function and making a quantitatively controlled change in $C$ is harmless.

We now state and prove two simple lemmas.

Lemma 2.2. A continuous function $g:[0, \infty) \rightarrow(0, \infty)$ is a sphericalizing function if and only if it satisfies (S1) and (S3), with quantitative control of parameters.

Proof. By integration, (S3) immediately implies (S2), with $C=C^{\prime} / \epsilon$. Conversely suppose (S1) and (S2) hold. Since $g(t) \geq g(r) / C$ for $r \leq t \leq 2 r$, we get the useful estimate

$$
\frac{r g(r)}{C} \leq \int_{r}^{\infty} g(t) d t \leq \operatorname{Crg}(r), \quad r \geq 1 .
$$

Writing $u(r):=r^{1 / C} \int_{r}^{\infty} g(t) d t$, we see that for all $r>1$,

$$
u^{\prime}(r)=\frac{r^{-1+1 / C}}{C}\left(\int_{r}^{\infty} g(t) d t-C r g(r)\right) \leq 0 .
$$

Thus $u$ is a decreasing function and

$$
\int_{s}^{\infty} g(t) d t \leq\left(\frac{r}{s}\right)^{1 / C} \int_{r}^{\infty} g(t) d t, \quad 1 \leq r \leq s .
$$

This inequality plus (2.3) gives (S3) with $C^{\prime}=C^{2}$ and $\epsilon=1 / C$.

Lemma 2.4. Suppose $g$ is a $C$-sphericalizing function, $C>2$. Then for every $K>0$ there exists $\alpha=\alpha(C, K) \in(0,1)$ such that

$$
\int_{\alpha r}^{r} g(t) d t \geq \operatorname{Krg}(r), \quad r \geq 1 / \alpha .
$$


Proof. Replacing $g(t)$ by the lower bound $g(r)(r / t)^{1+\epsilon} / C^{\prime}$ provided by Lemma 2.2, and integrating, we get

$$
\int_{\alpha r}^{r} g(t) d t \geq r g(r) \cdot\left(\frac{\alpha^{-\epsilon}-1}{\epsilon C^{\prime}}\right) .
$$

Taking $\alpha>0$ small enough, the parenthesized expression can be made arbitrarily large.

We record the following change of variables lemma, which is essentially a special case of $[\mathrm{V}$, Theorem 5.7].

Lemma 2.5. Let $I \subset[0, \infty)$ be an interval. If $\alpha:[a, b] \rightarrow I$ satisfies the 1-Lipschitz condition $|\alpha(s)-\alpha(t)| \leq|s-t|, s, t \in[a, b]$, and $\rho: I \rightarrow(0, \infty)$ is a Borel function, then

$$
\int_{a}^{b} \rho(\alpha(t)) d t \geq\left|\int_{\alpha(a)}^{\alpha(b)} \rho(s) d s\right| .
$$

We now come to the first of the three results in this section that require $d$ to be a length metric, and not just a local length metric.

Proposition 2.6. If $(X, d, o)$ is an unbounded pointed length space, and $\sigma=$ $\mathfrak{S}(d, o, g)$ for some $C$-sphericalizing function $g$, then $\operatorname{dia}_{\sigma}(X) \leq 4 C g(1)$.

Proof. Let $\gamma_{\epsilon} \in \Gamma_{d}(o, y)$ be a path of $d$-length $L \leq|y|+\epsilon$ for some $\epsilon \in(0,1]$, and so

$$
\operatorname{len}_{\sigma}(\gamma)=\int_{0}^{L} g\left(\left|\gamma_{\epsilon}(t)\right|\right) d t
$$

By the triangle inequality, $t-\epsilon \leq|\gamma(t)| \leq t$. Letting $\epsilon$ tend to zero, and using the uniform continuity of $g$ on $[0,|y|+1]$, we deduce that $\operatorname{len}_{\sigma}\left(\gamma_{\epsilon}\right)$ tends to $\int_{0}^{|y|} g(t) d t$. We claim that the last integral is bounded by $2 C g(1)$. To see this, estimate $\int_{0}^{1} g(t) d t$ and $\int_{1}^{\infty} g(t) d t$ using (S1) and (S2), respectively.

Remark 2.7. The above lemma would fail if we merely required $d$ to be a local length metric. For instance, if $d$ is the Euclidean metric on a domain $\Omega \subset \mathbb{R}^{n}$, then, using the standard sphericalizing function, $\sigma$ is the usual inner spherical metric, and this can be unbounded if $\Omega$ tightly spirals in the vicinity of some point $z \in \partial \Omega$. If we instead sphericalized $\Omega$ with respect to the inner Euclidean metric, we would always get a bounded space, although this space is typically not a subdomain of the Riemann sphere.

Remark 2.8. If $\gamma$ is a path in $X$ containing points $x, y$ with $|x| \leq|y|$, then

$$
\operatorname{len}_{\sigma}(\gamma) \geq \int_{|x|}^{|y|} g(t) d t
$$

This follows from Lemma 2.5 with $\rho=g$ and $\alpha(t)=|\nu(t)|$, where $\nu$ is the reparametrization of $\gamma$ by arclength. Since we already know from the proof of Proposition 2.6 that $\sigma(o, y) \leq \int_{0}^{|y|} g(t) d t$, it follows that $\sigma(o, y)=\int_{0}^{|y|} g(t) d t$, assuming $d$ is a length metric. 


\subsection{Escape properties.}

Definition 2.11. Suppose $(X, d, o)$ is a pointed locally complete local length space, $0<c \leq 1$, and $\rho \geq 0$. We say that $\gamma$ is a $c$-escape path for $x \in X$ if $\gamma:[0, T) \rightarrow X$ is parametrized by $d$-arclength, $\gamma(0)=x,|\gamma(t)| \geq c(t+|x|)$ for all $0 \leq t<T$, and either $T=\infty$, or $T<\infty$ with $\gamma(T-; d)=y \in \partial X_{l}$; recall that $\gamma(T-; d)$ indicates the $d$-limit $\lim _{t \rightarrow T^{-}} \gamma(t)$. We call $\gamma$ a strong c-escape path if $T=\infty$. We say that ( $X, d, o$ ) has the (strong) $(c, \rho)$-escape property if every $x \in X$ satisfying $|x| \geq \rho$ has a (strong) $c$-escape path.

Trivially, the strong $(c, \rho)$-escape property implies the $(c, \rho)$-escape property. By the triangle inequality, a $c$-escape path for $x$ must satisfy $|\gamma(t)| /(t+|x|) \in[c, 1]$. This implies the following fact.

Fact 2.12. If $g$ is a $C_{0}$-sphericalization function, and $\gamma:[0, T) \rightarrow X$ is a $c$ escape path for a point $x \in X$, then there exists $C=C\left(C_{0}, c\right) \geq 1$ such that $g(|\gamma(t)|) / g(t+|x|) \in[1 / C, C], 0 \leq t<T$.

Modulo a change in the values of $c$ and $\rho$, (strong) escape properties are independent of the basepoint $o$. Moreover the next lemma shows that if any point in a length space has a strong escape path, so does $o$.

Lemma 2.13. Let $(X, d, o)$ be an unbounded pointed local length space, and suppose that some point $x_{0} \in X$ has a strong c-escape path for some $0<c \leq 1$. Then o also has a strong $c^{\prime}$-escape path for all $0<c^{\prime}<c$.

Proof. We first claim that for all $0<\delta<1 / 4$ there is a path $\nu=\nu_{\delta}$ from $o$ to $x_{0}$, parametrized by $d$-arclength and satisfying $|\nu(t)| \geq(1-4 \delta) t$. We construct $\nu$ inductively. First pick a path $\lambda_{1}$ from $x_{0}$ to $o$ of length less than $(1+\delta)\left|x_{0}\right|$. Let $\nu_{1}$ be the initial segment of $\lambda_{1}$ as far as $x_{1}=\lambda_{1}\left(t_{1}\right)$, the first point on $\lambda_{1}$ whose distance from $o$ is $\left|x_{0}\right| / 2$. Inductively, given $x_{j}$, we find a path $\lambda_{j+1}$ from $x_{j}$ to $o$ of length less than $(1+\delta)\left|x_{j}\right|$ and let $\nu_{j+1}$ be the initial segment of $\lambda_{j+1}$ as far as $x_{j+1}=\lambda_{j+1}\left(t_{j+1}\right)$, the first point on $\lambda_{j+1}$ whose distance from $o$ is $\left|x_{j}\right| / 2$. Concatenating the paths $\nu_{j}, j \in \mathbb{N}$, and parametrizing the resulting path $\nu:[0, L] \rightarrow X$ by $d$-arclength in the direction from $o$ to $x_{0}($ with $\nu(0)=o$ ), it is clear that the resulting path $\nu$ justifies our claim.

Given $0<c^{\prime}<c$, suppose we want a strong $c^{\prime}$-escape path $\gamma$ for $o$. First let $\nu$ be as above, with $4 \delta=\left(1-c^{\prime} / c\right)$. Now let $\gamma$ be the concatenation of $\nu$ and the strong $c$-escape path $\lambda$ for $x_{0}$, parametrized by $d$-arclength. The desired lower bound for $|\gamma(t)|$ follows from the weaker inequality $1-4 \delta \geq c^{\prime}$ when $t \leq L:=\operatorname{len}_{d}(\nu)$, while for $t \geq L$, we have

$$
|\gamma(t)|=|\lambda(t-L)| \geq c\left(t-L+\left|x_{0}\right|\right) \geq c(t-L+(1-4 \delta) L) \geq c(1-4 \delta) t=c^{\prime} t .
$$

Example 2.14. Suppose that $\Omega \subset \mathbb{R}^{n}$ is a Euclidean domain containing the origin. Let $o$ be the origin and attach the inner Euclidean metric $d$. Then $\Omega$ has the $(1,0)$ escape property, since if $x \in \Omega, x \neq 0$, we can take the path $\gamma:[0, T) \rightarrow \Omega$, $\gamma(t)=x+t \cdot x$, with $T$ being the least value of $t>0$ for which $x+t \cdot x \in \partial \Omega_{d}$ (or $T=\infty$ if there is no such number $t$ ). If $x=0$, take any ray beginning at the origin, and let $\gamma$ be the initial part which ends (if at all) when this ray first reaches $\partial \Omega_{d}$. It is, however, easy to give an example of such a domain where $o$ (and hence 
every point) fails to have any strong escape paths, for instance the complement of an Archimidean spiral $S \not \supset o$.

Example 2.15. There are also domains in which there are strong escape paths from all points but which fail to have a strong escape property because the escape parameter $c$ depends on the point. Consider, for instance, the domain whose complement consists of the two curves given by $t \mapsto\left(t, 1+t^{2} \sin t\right), t \geq 0$, and $t \mapsto\left(t, 3+t^{2} \sin t\right), t \geq 0$. There is a strong escape path from every point $x \in \Omega$, but the strong escape parameter $c$ depends on $x$. For instance, if we write

$$
\Omega^{\prime}=\left\{\left(x_{1}, x_{2}\right): x_{1} \geq 0,\left|x_{2}-2-x_{1}^{2} \sin x_{1}\right|<1\right\} \subset \Omega,
$$

then for points in $\left(x_{1}, x_{2}\right) \in \Omega^{\prime}$, the parameter $c$ must be chosen to be very small if $x_{1}$ is very large. Thus $\Omega$ fails to have any strong escape property. By contrast, there is a strong 1-escape path from all points $\left(x_{1}, x_{2}\right)$ with $x_{1}<0$.

Example 2.16. Consider $X \subset \mathbb{R}^{2}$, where $X$ consists of the horizontal half-line $\left(x_{1}, 0\right), x_{1} \geq 0$, together with the attached vertical line segments $\left(a_{n}, x_{2}\right), 0 \leq$ $x_{2} \leq b_{n}, n \in \mathbb{N}$, where $\left(a_{n}\right)$ is a strictly increasing sequence of positive numbers tending to infinity, and $\left(b_{n}\right)$ is a nonnegative sequence of numbers. Let $o=(0,0)$ and equip $X$ with its natural Euclidean length metric $d$. Then it is readily verified that $(X, d, o)$ has the $(c, \rho)$-escape property for a particular $0<c \leq 1$ if and only if $b_{n} \leq(1-c) a_{n} / 2 c$ whenever $a_{n}+b_{n} \geq \rho$. In particular, $(X, d, o)$ has some escape property if and only if $\left(b_{n} / a_{n}\right)_{n=1}^{\infty}$ is bounded. The same condition also characterizes when $(X, d, o)$ has a strong escape property.

Example 2.17. Suppose $(X, d, o)$ is as in the previous example, with $b_{n}>0$ for all $n \in \mathbb{N}$. Given $T \subset \mathbb{N}$, we define $X_{T}$ to be the subset of $X$ consisting of all points except $\left(a_{n}, b_{n}\right), n \in T$, and let us also denote by $d$ the subspace metric inherited by $X_{T}$. Then $\left(X_{T}, d, o\right)$ is always a length space, and it has an escape property if and only if the set $\left\{b_{n} / a_{n}: n \in \mathbb{N} \backslash T\right\}$ is bounded. In the extreme case $T=\mathbb{N}$, $\left(X_{\mathbb{N}}, d, o\right)$ always satisfies the $(1,0)$-escape property. Notice however that strong escape properties for spaces of the form $\left(X_{T}, d, o\right)$ are more restrictive than escape properties whenever $T$ is nonempty; although, there always exists a strong escape path for $o$ and all other points $\left(x_{1}, 0\right) \in X_{T}$, nevertheless $\left(X_{T}, d, o\right)$ fails to satisfy a strong escape property unless the full sequence $\left(b_{n} / a_{n}\right)_{n=1}^{\infty}$ is bounded.

Example 2.18. Let $X$ consist of a bouquet of half-open line segments $I_{n}$, where $I_{n}$ has length $n$. Here, we identify the included endpoints of all the intervals and call this point $o$. There is a natural length metric $d$ on $X$ which restricts to the length metric on each interval $I_{n}$. Then $(X, d, o)$ satisfies a $(1,0)$-escape property, but there is no strong escape path from any point of $X$.

2.19. The spherical boundary. Suppose $\sigma=\mathfrak{S}(d, o, g)$, where $g$ is a sphericalizing function. Given $x \in X, y \in \partial X_{l}$, and $\gamma \in \Gamma_{d}(x, y)$, it is clear that $\gamma$ is also of finite $\sigma$-length. Thus we can, and will, view $\partial X_{l}$ as a subset of $\partial X_{\sigma}$. We define the spherical boundary of $X$ to be $\partial_{\infty} X:=\partial X_{\sigma} \backslash \partial X_{l}$. It is clear that if $x \in X$, $y \in \partial_{\infty} X$, and $\gamma \in \Gamma_{\sigma}(x, y)$, then $\gamma$ must exit from all balls $B_{d}(o, r)$. Thus $\partial_{\infty} X$ is a type of "boundary at infinity" for the space $(X, d)$ which generalizes the role of the point at infinity on the Riemann sphere. 
Proposition 2.20. Suppose $(X, d, o)$ is an unbounded pointed local length space and $\sigma=\mathfrak{S}(d, o, g)$ for some $C$-sphericalizing function $g$. If some point $x \in X$ has a strong escape path, then $\partial_{\infty} X$ is nonempty. In any case, $\partial_{\infty} X$ is always a closed subset of $\bar{X}_{\sigma}$.

Proof. Let $\gamma:[0, \infty) \rightarrow X$ be a strong escape path, so that $\gamma$ is parametrized by $d$-arclength, $\gamma(0)=x$, and $|\gamma(t)| \geq c(t+|x|)$. Using Fact 2.12, (S1) and (S2), we get

$$
\begin{aligned}
\operatorname{len}_{\sigma}(\gamma) & =\int_{0}^{\infty} g(|\gamma(t)|) d t \approx \int_{0}^{\infty} g(t+|x|) d t \\
& =\int_{|x|}^{\infty} g(s) d s \lesssim(1+|x|) g(|x|) .
\end{aligned}
$$

Since $\gamma$ has finite $\sigma$-length, $\gamma(t)$ has a $\sigma$-limit $w \in \bar{X}_{\sigma}$ as $t \rightarrow \infty$. Now $w$ cannot be in $\bar{X}_{l}$, since then $\gamma$ would have to $d$-converge to $w$. Thus $w \in \partial_{\infty} X$, and $\partial_{\infty} X$ is nonempty.

Since paths converging to $\partial_{\infty} X$ must leave all balls $B_{d}(o, r)$, it follows from (2.9) that

$$
\operatorname{dist}_{\sigma}\left(y, \partial_{\infty} X\right) \geq \int_{|y|}^{\infty} g(t) d t, \quad y \in \bar{X}_{l} .
$$

So a sequence in $\partial_{\infty} X$ cannot converge to a point in $\bar{X}_{l}$, and $\partial_{\infty} X$ is $\sigma$-closed.

Example 2.23. In the absence of the strong escape path assumption, $\partial_{\infty} X$ might be empty. This is indeed the case if $X=\bigcup_{j=1}^{\infty} S_{j}$, where $S_{j} \subset \mathbb{R}^{2}$ is the circle centered at $(j, 0)$ of radius $j$; as usual, $d$ is the inner Euclidean metric and $o$ is the origin. Then $\partial X_{l}$ and $\partial_{\infty} X$ are both easily seen to be empty. For the latter fact, suppose we have a sequence $\left(x_{n}\right)$ in $X$ satisfying $1 \leq\left|x_{n}\right| \rightarrow \infty$. It is clear that if we define $j_{n}$ by the equation $x_{n} \in j_{n}$, then $j_{n} \rightarrow \infty$ as $n \rightarrow \infty$. In particular for each $n \in \mathbb{N}$, we can find $m>n$ such that $j_{m} \neq j_{n}$, and so by (S1) and (2.9) we get the estimate $\sigma\left(x_{n}, x_{m}\right) \geq 2 g(1) / C>0$. Thus $\left(x_{n}\right)$ is not Cauchy in $(X, \sigma)$.

A similar example is the space $X$ in Example 2.18; there $\partial_{\infty} X$ is empty, but $\partial X_{l}$ is nonempty.

2.24. Comparability of quasihyperbolic metrics. Let us first introduce some notation and estimates that will be used throughout the remainder of this section. Suppose $g$ is a $C$-sphericalizing function. Given $x \in X$, let $B_{x}=B_{d}\left(x, r_{x}\right)$, where $r_{x}:=1 \vee(|x| / 2)$. Let $A_{j}=B_{d}\left(o, 2^{j}\right) \backslash B_{d}\left(o, 2^{j-1}\right), j \in \mathbb{N}, A_{0}=B_{d}(o, 1)$, and let $A_{-1}$ be the empty set. We also define fatter annuli $A_{i, j}=\bigcup_{m=i}^{j} A_{m}$ for all $-1 \leq i \leq j \leq \infty$. We denote the $d$-closure of any of these sets by means of a bar: $\bar{B}_{x}, \bar{A}_{j}$, etc. Note that $B_{x} \subset A_{j-1, j+1}$ for all $x \in A_{j}$.

It follows from (S1) that $g(|y|) / g(|x|) \in[1 / C, C]$ for all $y \in \bar{B}_{x}$, and that $g(|y|) / g(|z|) \in[1 / C, C]$ for all $y, z \in \bar{A}_{j}$. Thus if $\gamma$ is a path in $X$, then

$$
\begin{aligned}
C^{-1} & \leq \frac{\operatorname{len}_{\sigma}(\gamma)}{\operatorname{len}_{d}(\gamma) g(|x|)} \leq C, \quad \gamma \subset \bar{B}_{x}, \\
C^{i-j-1} & \leq \frac{\operatorname{len}_{\sigma}(\gamma)}{\operatorname{len}_{d}(\gamma) g(|x|)} \leq C^{j+1-i}, \quad \gamma \subset \bar{A}_{i, j}, x \in \bar{A}_{i, j}, 0 \leq i \leq j .
\end{aligned}
$$


If $\gamma$ is a path that passes through $x$ of length at least $r_{x}$, then it has a piece of length at least $r_{x}$ inside $B_{x}$, and so (2.25) implies that

$$
\operatorname{len}_{\sigma}(\gamma) \geq C^{-1} r_{x} g(|x|) .
$$

In particular, this estimate holds for any path through $x$ that is not contained in $B_{x}$, and for any path through $x \in \bar{A}_{i}$ that is not contained in $A_{i-1, i+1}$.

We now state and prove the main result of this section which concerns the relationship between quasihyperbolic metrics associated with $d$ and $\sigma$. We do not want to restrict $(X, d)$ to be incomplete, so we use $k_{d, C}$ for some $C<\infty$ as the quasihyperbolic metric associated with $d$. By contrast, only incomplete spaces $(X, \sigma)$ are of interest, so we use $k_{\sigma}$ as the quasihyperbolic metric associated with $\sigma$. Note that

$$
\begin{aligned}
k_{d, C}(x, y) & =\inf _{\gamma \in \Gamma(x, y ; d)} \int_{\gamma} \frac{d l(z)}{\delta_{d, C}(z)}, \\
k_{\sigma}(x, y) & =\inf _{\gamma \in \Gamma(x, y ; \sigma)} \int_{\gamma} \frac{d \sigma(z)}{\delta_{\sigma}(z)}=\inf _{\gamma \in \Gamma(x, y ; \sigma)} \int_{\gamma} \frac{g(|z|) d l(z)}{\delta_{\sigma}(z)},
\end{aligned}
$$

where $d l$ and $d \sigma$ are the length elements with respect to $d$ and $\sigma$, respectively. Note also that if $(X, d)$ is locally incomplete, then so is $(X, \sigma)$.

Theorem 2.28. Let $(X, d, o)$ be a locally complete unbounded pointed local length space, let $\sigma:=\mathfrak{S}(d, o, g)$ for some $C_{g}$-sphericalizing function $g$, and let $1 \leq C_{d}<\infty$.

(a) If $\partial X_{\sigma}$ is nonempty, then $k_{\sigma} \leq C^{\prime} k_{d, C_{d}}$, where $C^{\prime}=C^{\prime}\left(C_{g}, C_{d}\right)$.

(b) If $(X, d, o)$ has some $(c, \rho)$-escape property, then $\partial X_{\sigma}$ is nonempty and $k_{\sigma} \geq$ $c^{\prime} k_{d, C_{d}}$ for some $c^{\prime}=c^{\prime}\left(c, \rho, C_{g}\right)>0$.

(c) Conversely, if $k_{\sigma} \geq c^{\prime} k_{d, C_{d}}$ for some $c^{\prime}>0$, then $(X, d, o)$ has a $(c, \rho)$-escape property, for some $c, \rho$ dependent only on $c^{\prime}, C_{g}$, and $C_{d}$.

Proof. Since $k_{d, C_{d}}$ is a conformal deformation of $d$ with density function $1 / \delta_{d, C_{d}}(x)$, and $k_{\sigma}$ is a conformal deformation of $d$ with density function $g(|x|) / \delta_{\sigma}(x)$, inequalities between $k_{d, C_{d}}$ and $k_{\sigma}$ are equivalent to the corresponding reverse inequalities between $\delta_{\sigma}(x)$ and $g(|x|) \delta_{d, C_{d}}(x)$.

To prove (a), we need to prove that $\delta_{\sigma}(x) \gtrsim g(|x|) \delta_{d, C_{d}}(x)$. Thus we need to find $C^{\prime}=C^{\prime}\left(C_{g}, C_{d}\right)$ such that for all $y \in \partial X_{\sigma}, \gamma \in \Gamma_{\sigma}(x, y)$,

$$
\operatorname{len}_{\sigma}(\gamma) \geq g(|x|) \delta_{d, C_{d}}(x) / C^{\prime} .
$$

Let $j \geq 0$ be such that $x \in A_{j}$, and let $\gamma^{-}$be the half-open curve $\gamma \backslash\{y\}$. If $\gamma^{-} \subset A_{j-1, j+1}$, then necessarily $y \in \partial X_{l}$ and so $(2.26) \operatorname{implies}$ that $\operatorname{len}_{\sigma}(\gamma) \geq$ $g(|x|) \operatorname{len}_{d}(\gamma) / C_{g}^{3} \geq g(|x|) \delta_{d, C_{d}}(x) / C_{g}^{3}$. On the other hand, if $\gamma^{-} \not \subset A_{j-1, j+1}$, then by $(2.27), \operatorname{len}_{\sigma}\left(\gamma \cap A_{j-1, j+1}\right) \gtrsim g(|x|) 2^{j}$. But $\delta_{d, C_{d}}(x) \leq C_{d}+|x| \lesssim 2^{j}$, so again $\operatorname{len}_{\sigma}(\gamma) \gtrsim g(|x|) \delta_{d, C_{d}}(x)$.

We now turn to the proof of (b). Suppose that $(X, d, o)$ has the $(c, \rho)$-escape property; we assume without loss of generality that $\rho \geq 2$. We must prove that $\delta_{\sigma}(x) \lesssim g(|x|) \delta_{d, C_{d}}(x)$. If $\delta_{d, C_{d}}(x)<r_{x}$, then $\delta_{d, C_{d}}(x)=\delta_{d}(x)$, so the local length property ensures that we can choose $y \in \partial X_{l}$ and $\gamma \in \Gamma_{d}(x, y)$ with $L:=\operatorname{len}_{d}(\gamma)<$ $r_{x} \wedge\left(2 \delta_{d}(x)\right)$. Now $(2.25)$ tells us that

$$
\delta_{\sigma}(x) \leq \operatorname{len}_{\sigma}(\gamma) \leq C_{g} g(|x|) L \leq 2 C_{g} g(|x|) \delta_{d, C_{d}}(x) .
$$

We may therefore assume that $\delta_{d, C_{d}}(x) \geq r_{x} \geq 1$. 
Suppose $|x| \geq \rho$. Let $\gamma:[0, T) \rightarrow X$ be parametrized by $d$-arclength such that $\gamma(0)=x,|\gamma(t)| \geq c(t+|x|)$, and either $T=\infty$, or $T<\infty$ with $\gamma(T-; d)=y \in \partial X_{l}$. Now $|x| \geq \rho \geq 2$ and $r_{x}=|x| / 2 \leq \delta_{d, C_{d}}(x)$, so if $T<\infty$, then $y \in \partial X_{l} \subset \partial X_{\sigma}$, so $\partial X_{\sigma}$ is nonempty. Furthermore, using Fact 2.12 and (S2), we get

$$
\delta_{\sigma}(x) \leq \int_{0}^{T} g(|\gamma(t)|) d t \approx \int_{0}^{T} g(t+|x|) d t \leq C_{g} g(|x|)|x| \leq 2 C_{g} g(|x|) \delta_{d, C_{d}}(x),
$$

as desired. If instead $T=\infty$, it follows similarly that $\operatorname{len}_{\sigma}(\gamma) \lesssim g(|x|)|x|$, and so $\gamma(t)$ is $\sigma$-convergent to some point $w \in \bar{X}_{\sigma}$. But $w$ cannot be in $X \cup \partial X_{l}$, since $\gamma$ would then have to $d$-converge to $w$. Thus $w \in \partial_{\infty} X$, so $\partial X_{\sigma}$ is again nonempty and $\delta_{\sigma}(x) \leq \operatorname{len}_{\sigma}(\gamma) \lesssim g(|x|) \delta_{d, C_{d}}(x)$.

Suppose instead that $|x|<\rho$. Since $X$ is an unbounded local length space, there exists $x_{0} \in X$ with $\left|x_{0}\right|=\rho$. By the previous case with $C_{d}=1$, we see that $\delta_{\sigma}\left(x_{0}\right) \lesssim g(\rho)(1+\rho) \lesssim g(|x|)$. If $|x|+d\left(x, x_{0}\right) \leq \delta_{d}(x)$, then, as in the proof of Proposition 2.6, we see that $\sigma\left(x, x_{0}\right) \leq 4 C g(1) \lesssim g(|x|)$, and so

$$
\delta_{\sigma}(x) \leq \sigma\left(x, x_{0}\right)+\delta_{\sigma}\left(x_{0}\right) \lesssim g(|x|) \lesssim g(|x|) \delta_{d, C_{d}}(x) .
$$

Otherwise, we take a path $\gamma \in \Gamma(x, y ; d), y \in \partial X_{l}$, satisfying $\operatorname{len}_{d}(\gamma)<|x|+d\left(x, x_{0}\right)$ $<3 \rho$ and so

$$
\delta_{\sigma}(x) \leq \sigma(x, y) \lesssim g(|x|) \lesssim g(|x|) \delta_{d, C_{d}}(x) .
$$

This finishes the proof of (b).

Finally we prove (c). Assume that $\delta_{\sigma}(z) \leq g(|z|) \delta_{d, C_{d}}(z) / c^{\prime}, z \in X$. We also assume without loss of generality that $0<c^{\prime}<1$. Let $K=2\left(C_{d}+1\right) / c^{\prime}$ and $\rho=\alpha^{-1}>1$, where $\alpha=\alpha\left(C_{g}, K\right)$ is as in Lemma 2.4. We prove that $(X, d, o)$ has a $(c, \rho)$-escape property for some $0<c<1$ by constructing an escape path $\gamma$ for an arbitrary point $x,|x| \geq \rho$. Let $\lambda_{0}:\left[0, T_{0}\right) \rightarrow X$ be a path parametrized by $\sigma$-arclength such that $\lambda_{0}(0)=x, \lambda_{0}\left(T_{0}-; \sigma\right)=w_{0} \in \partial X_{\sigma}$, and $T_{0}<2 g(|x|) \delta_{d, C_{d}}(x) / c^{\prime}$. Let $\nu_{0}:\left[0, S_{0}\right) \rightarrow X$ be the reparametrization of $\lambda_{0}$ by $d$-arclength. Note that $S_{0}$ may be finite or infinite.

We first show that $\lambda_{0}$ stays outside the ball $B_{d}(o,|x| / \rho)$. If this were not the case, then according to Lemma 2.4, we would have

$$
T_{0}=\operatorname{len}_{\sigma}\left(\lambda_{0}\right)>\int_{|x| / \rho}^{|x|} g(t) d t>K|x| g(|x|) .
$$

Since $\delta_{d, C_{d}}(x) \leq\left(C_{d}+1\right)|x|$, this would contradict the fact that

$$
T_{0}<2 g(|x|) \delta_{d, C_{d}}(x) / c^{\prime} \leq K|x| g(|x|) .
$$

Let $s_{1}$ be the least number $s$ for which $\left|\nu_{0}(s)\right|=2|x|$, or let $s_{1}=S_{0}$ if $\left|\nu_{0}(s)\right|<$ $2|x|$ for all $0 \leq s<S_{0}$. We define $x_{1}=\nu_{0}\left(s_{1}\right)$ and $\gamma_{1}=\left.\nu_{0}\right|_{\left[0, s_{1}\right]}$. Since the values $g(t), t \in[|x| / \rho, 2|x|]$, are all mutually comparable, the bound $T_{0}<K|x| g(|x|)$ implies that $s_{1} \lesssim|x|$. If $s_{1}=S_{0}$, we have reached a stopping point and $\gamma_{1}$ is the desired escape path; notice that $\left|\gamma_{1}(t)\right| \geq|x| / \rho$ for $t \in\left[0, s_{1}\right]$. Otherwise we iterate the argument as follows.

We choose a path $\lambda_{1}:\left[0, T_{1}\right) \rightarrow X$, parametrized by $\sigma$-arclength, such that $\lambda_{1}(0)=x_{1}, \lambda_{1}\left(T_{1}-; \sigma\right)=w_{1} \in \partial X_{\sigma}$, and $T_{1}<2 g\left(\left|x_{1}\right|\right) \delta_{d, C_{d}}\left(x_{1}\right) / c^{\prime}$. Let $\nu_{1}$ : $\left[0, S_{1}\right) \rightarrow X$ be the reparametrization of $\lambda_{1}$ by $d$-arclength. Replacing the input data $\left(x, w_{0}, \lambda_{0}, \nu_{0}\right)$ in the above argument by $\left(x_{1}, w_{1}, \lambda_{1}, \nu_{1}\right)$, we get as output $\left(s_{2}, x_{2}, \gamma_{2}\right)$ where $s_{2} \lesssim\left|x_{1}\right|, x_{2}=\nu_{1}\left(s_{1}\right)$, and $\gamma_{2}=\left.\nu_{1}\right|_{\left[0, s_{2}\right]}$. Furthermore, either 
$s_{2}=S_{1}$, in which case we have reached a stopping point, or $s_{2}<S_{1}$ and $\left|x_{2}\right|=2\left|x_{1}\right|$, in which case we continue to iterate the process.

We continue this iterative procedure until we reach a stopping point, or forever if this never occurs. All the paths $\gamma_{j}$ obtained by this process can be joined to form a single path $\gamma$ parametrized by $d$-arclength. Since each $\gamma_{j}$ has $d$-arclength $s_{j} \lesssim 2^{j}$, and each point $y \in \gamma_{j}$ has the property that $|y| \approx 2^{j}|x|$, it readily follows that $\gamma$ is an escape path for $x$.

Note that if $(X, d)$ is incomplete, then $\delta_{d, C}=\delta_{d}$ and $k_{d, C}=k_{d}$ for all $C \geq$ $d\left(o, \partial X_{d}\right)$. Thus Theorem 2.28 says that, in the presence of an escape property, the usual quasihyperbolic metrics $k_{d}$ and $k_{\sigma}$ are comparable with a constant dependent only on the escape parameters, $C_{g}$, and $d(o, \partial X)$.

Since Euclidean domains are locally complete and satisfy a $(1,0)$-escape property, we can, as a special case, apply this last statement with $(X, d)$ being the Euclidean domain $\Omega \subsetneq \mathbb{R}^{n}, g(t)=2 /\left(1+t^{2}\right)$, and $o=x_{0} \in \Omega$. The resulting sphericalized metric $\sigma$ is a bilipschitz distortion of the usual inner spherical metric $\sigma^{\prime}$, with a bilipschitz constant dependent only on $\left|x_{0}\right|$; note that $\sigma=\sigma^{\prime}$ only if $x_{0}=0$; note that the case $n=1$ is a little different, but the result is still valid in this case. Thus we have proven Theorem 0.1 .

2.30. Reverse escape properties. In $\S 3.4$, we will discuss the concept of (strong) reverse escape properties in more detail but for now we wish to show only that the sphericalization process always produces data with such a property. Given $\sigma:=\mathfrak{S}(d, o, g)$ as usual, and $0<c \leq 1$, we say that $(X, \sigma, o)$ has the reverse $c$ escape property if, for every $x \in X$, there exists $y \in\{o\} \cup \partial X_{l}$ and $\gamma \in \Gamma_{\sigma}(x, y)$ such that $\delta_{\infty}(\gamma(t)) \geq c\left(t+\delta_{\infty}(x)\right), 0 \leq t \leq T=\operatorname{len}_{\sigma}(\gamma)$, where $\delta_{\infty}(z):=\operatorname{dist}_{\sigma}\left(z, \partial_{\infty} X\right)$. If we can always take $y=o$ in this condition, we say that $(X, \sigma, o)$ has the strong reverse c-escape property. Note that the inequality $\delta_{\infty}(\gamma(t)) \geq\left(t+\delta_{\infty}(x)\right) / 4$ holds for all $\gamma \in \Gamma_{\sigma}(x, y)$ when $0 \leq t \leq \delta_{\infty}(x) / 2$. To prove a (strong) reverse escape property, we need only show that $\delta_{\infty}(\gamma(t)) \gtrsim t$ when $t \geq \delta_{\infty}(x) / 2$.

Theorem 2.31. Let $(X, d, o)$ be a pointed local length space, and let $\sigma=\mathfrak{S}(d, o, g)$, for some $C_{g}$-sphericalizing function $g$. Then

(a) $(X, \sigma, o)$ has a reverse c-escape property, and

(b) if $d$ is a length metric, then $(X, \sigma, o)$ has a strong reverse c-escape property. Moreover, $c>0$ can be taken to depend only on $C_{g}$.

Proof. Let us prove (b). We need to find a strong reverse escape path $\gamma$ for a general point $x \in X$. If $|x| \leq 1$, we can take $\gamma \in \Gamma(x, o ; d)$ to be any path of $d$-length at most 2: such a path must remain in the ball $\bar{B}_{d}(o, 2)$, and so using (S1) we get $T:=\operatorname{len}_{\sigma}(\gamma) \lesssim g(1)$. Similarly, if $z, z^{\prime} \in \bar{B}_{d}(0,2)$, then $\left|\delta_{\infty}(z)-\delta_{\infty}\left(z^{\prime}\right)\right| \leq$ $\sigma\left(z, z^{\prime}\right) \lesssim g(1)$. But by (2.22), (S1), and (S2), we have $\delta_{\infty}(z) \gtrsim \int_{2}^{\infty} g(t) d t \gtrsim g(1)$ whenever $z \in \bar{B}_{d}(0,2)$. Thus for $0 \leq t \leq T$, we have $\delta_{\infty}(\gamma(t)) \approx \delta_{\infty}(x) \gtrsim g(1) \gtrsim t$, thus giving the desired strong reverse escape property.

We may therefore assume that $|x|>1$. For $z \in X$, define $j(z)$ to be the nonnegative integer satisfying $z \in A_{j(z)}$. Let $\gamma \in \Gamma_{\sigma}(o, x)$ have $d$-length $L$ and $\sigma$ length $M$, where $L \leq|x|+1$. We cut $\gamma$ into segments $\gamma_{i}=\left.\gamma\right|_{\left[s_{i-1}, s_{i}\right)}$, where $s_{0}=0$, $s_{j(x)}=M$ and, for $0<i<j(x), \gamma\left(s_{i}\right)$ is the last point on $\gamma$ contained in $\overline{A_{i}}$. By construction, $\gamma_{i} \subset A_{i-1, i+1}$ and $\operatorname{len}_{d}\left(\gamma_{i}\right) \leq 2^{i}+1 \lesssim 2^{i}$. Thus $\operatorname{len}_{\sigma}\left(\gamma_{i}\right) \lesssim 2^{i} g\left(2^{i}\right)$. 
But by (S3), the series $\sum_{i=j(w)}^{\infty} 2^{i} g\left(2^{i}\right)$ is dominated by a convergent geometric series, and so $M-t \lesssim 2^{j(w)} g\left(2^{j(w)}\right)$ whenever $w=\gamma(t)$.

If we prove the estimate $\delta_{\infty}(w) \gtrsim 2^{j(w)} g\left(2^{j(w)}\right)$, it therefore follows that the reverse parametrization $-\gamma \in \Gamma_{\sigma}(x, o)$ is a strong reverse escape path. But this last estimate follows rather easily. Indeed if $\lambda$ is a path from $w$ to $\partial_{\infty} X$, then $(2.22),(\mathrm{S} 1)$, and (S2) tell us that

$$
\operatorname{len}_{\sigma}(\lambda) \geq \int_{|w|}^{2|w|+1} g(t) d t \gtrsim(|w|+1) g(|w|) .
$$

Part (a) follows like (b) if $\delta_{d}(x) \geq|x|$. Otherwise we argue in a similar fashion, but using a path $\gamma \in \Gamma_{\sigma}(y, x)$ of length at most $\delta_{d}(x)+1$, where $y \in \partial X_{l}$.

We cannot conclude in Theorem 2.31(a) that $(X, \sigma, o)$ has a strong reverse $c$ escape property, as the following example indicates.

Example 2.32. Consider the domain $X \subset \mathbb{R}^{2}$ given by the curve complement

$$
\left\{(s, t) \mid s, t \in \mathbb{R}, s \geq 1, t \neq s^{2} \sin ^{2} s\right\} \subset \mathbb{R}^{2} .
$$

Taking $d$ to be the Euclidean metric, $o=(0,0)$, and $g(t)=2 /\left(1+t^{2}\right)$, the metric $\sigma=\mathfrak{S}(d, o, g)$ is the inner spherical metric. The space $(X, \sigma, o)$ fails to have any strong reverse $c$-escape property, as we see by taking $x=(j \pi, 1) \in X$ for sufficiently large $j=j_{c} \in \mathbb{N}$.

\section{Flattening}

In this section we proceed in a direction opposite to that of the last section: We "flatten" a bounded incomplete local length space $(X, \tau)$ to get an unbounded length space $(X, l)$. We use the term "flattening" because this process generalizes that of obtaining Euclidean space from the Riemann sphere. The main result, Theorem 3.11, compares the corresponding quasihyperbolic metrics $k_{\tau}$ and $k_{l, C}$. For a basic example of this process, let $(X, \tau)$ be a domain on the Riemann sphere $\overline{\mathbb{R}^{n}}$ with $\infty \in \partial \Omega_{\tau}$. Then $l$ is the inner Euclidean metric on $\Omega$ (if we make the standard choice of flattening function $f$ and spherical boundary $\{\infty\}$ ). Alternatively, we can obtain the Euclidean metric on $\Omega$ by flattening $\overline{\mathbb{R}^{n}} \backslash\{\infty\}$ and restricting the resulting metric $l$ to $\Omega$.

Intuitively we think of flattening as being a concept roughly dual to the sphericalization, and this guides our reuse of much of the notation of Section 2. However we warn the reader that the concepts defined here, and their inter-relationships, are not necessarily the same as those in Section 2. We discuss some of the immediate parallels between the two approaches as we go along, but the deeper questions about when these two constructions are genuinely dual are postponed until Section 4.

Throughout this section, we assume that the data $\left(X, \tau, \partial_{\infty} X\right)$ are $d_{0}$-spherical data for some $0<d_{0}<\infty$, which we define to mean that the data have the following pair of properties:

(1) $(X, \tau)$ is an incomplete local length space of diameter at most $d_{0}$.

(2) $\partial_{\infty} X$ is a nonempty closed subset of $\bar{X}_{\tau}$.

We write $\delta_{\infty}(x)=\tau\left(x, \partial_{\infty} X\right)$, for all $x \in \bar{X}_{\tau}$.

We denote by $\sigma$ the inner metric associated with $\tau$. We will use $\sigma$ only in two contexts: the length element $d \sigma$ associated with $\tau$, and the $\sigma$-boundary $\partial X_{\sigma}$. We write $\delta_{\infty}(x)=\tau\left(I(x), \partial_{\infty} X\right)$ for all $x \in \partial X_{\sigma}$ where $I: \partial X_{\sigma} \rightarrow \partial X_{\tau}$ is the natural 
injection as in $\S 1.2$. We denote by $\partial_{\text {ns }} X$ the part of the $\sigma$-boundary that does not correspond to $\partial_{\infty} X$, i.e. $\partial_{\mathrm{ns}} X=\partial X_{\sigma} \backslash I^{-1}\left(\partial_{\infty} X\right)$; note that if $\tau$ is a length metric, then $\partial_{\mathrm{ns}} X=\partial X_{\tau} \backslash \partial_{\infty} X$.

By the results of Section 2, we know that if we sphericalize a length space in which $o$ has a strong escape path, we get spherical data $\left(X, \tau, \partial_{\infty} X\right)$, with $\tau:=\sigma$ being a length metric. However, we will see in the next section that there are such spherical data that do not arise in this way, even modulo a bilipschitz distortion.

Fixing a function $f:\left(0, d_{0}\right] \rightarrow(0, \infty)$, we define a new metric $\mathfrak{L}\left(\tau, \partial_{\infty} X, f\right)$ on $X$ by the equation

$$
\mathfrak{L}\left(\tau, \partial_{\infty} X, f\right)(x, y)=\inf _{\gamma \in \Gamma(x, y ; \tau)} \int_{\gamma} f\left(\delta_{\infty}(z)\right) d \sigma(z), \quad x, y \in X .
$$

We normally write $l$ in place of $\mathfrak{L}\left(\tau, \partial_{\infty} X, f\right)$, and we take $f$ to be any flattening function, a concept we now define.

3.1. Flattening functions. A continuous function $f:\left(0, d_{0}\right] \rightarrow(0, \infty)$ is a $\left(C, d_{0}\right)$-flattening function, $C>2, d_{0}>0$, if it has the following properties:

(F1) $f(r) \leq C f(s)$ whenever $r, s \in\left(0, d_{0}\right], 1 / 2 \leq r / s \leq 2$;

(F2) $\int_{r}^{d_{0}} f(t) d t \leq C r f(r)$, whenever $0<r \leq d_{0}$.

Typical flattening functions include $f(t)=\log ^{q}(2+1 / t) t^{-p}$, for $p>1, q \in \mathbb{R}$. The usual flattening of the Riemann sphere into the Euclidean metric uses $f(t)=$ $\csc ^{2}(t / 2) / 2,0<t \leq d_{0}:=\pi$; note that $f$ is comparable with $t \mapsto t^{-2}$.

It follows from (F2) that if $f$ is a flattening function, then $f(r) \geq c / r$ for all $0<r<d_{0} / 2$, where $c=C^{-1} \int_{d_{0} / 2}^{d_{0}} f(t) d t$. Consequently, $\int_{0}^{d_{0}} f(t) d t=\infty$; the divergence of this integral pushes $\partial_{\infty} X$ "infinitely far" from every point in $X$ with respect to the metric $l=\mathfrak{L}\left(\tau, \partial_{\infty} X, f\right)$. Consequently, we see that we can naturally identify $\partial X_{l}$ with $\partial_{\mathrm{ns}} X$. In particular, if $\tau$ is a length metric, we can identify $\partial X_{\tau}$ with the disjoint union of $\partial_{\infty} X$ and $\partial X_{l}$; this of course mirrors the relationship between the corresponding types of boundary in Section 2 .

We now state two lemmas. The first gives a useful alternative description of flattening functions, and it readily implies the second lemma. We omit both proofs, as they are very similar to those of Lemmas 2.2 and 2.4.

Lemma 3.2. A Borel function $f:\left(0, d_{0}\right] \rightarrow(0, \infty)$ is a flattening function if and only if it satisfies (F1) and a condition of the following type for some constants $C^{\prime} \geq 1, \epsilon>0$ :

$$
\text { (F3) } \frac{f(r)}{f(s)} \leq C^{\prime}\left(\frac{s}{r}\right)^{1+\epsilon} \text {, for all } 0<s \leq r \leq d_{0} .
$$

Furthermore, this equivalence holds with quantitative control of parameters.

Lemma 3.3. Given $C>2$ and $d_{0}, K>0$, there exists $\alpha=\alpha(C, K)$ such that, whenever $f$ is a $C$-flattening function and $0<r \leq d_{0}$,

$$
\int_{\alpha r}^{r} f(t) d t \geq K r f(r)
$$

We will see that the quasihyperbolic metrics associated with $(X, \tau)$ and $(X, l)$ are bilipschitz equivalent if and only $(X, \tau, o)$ satisfies a reverse escape property similar to the one at the end of Section 2. 
3.4. Reverse escape properties revisited. Suppose $\left(X, \tau, \partial_{\infty} X\right)$ are spherical data. Given $0<c \leq 1$, we say that $(X, \tau, o)$ has the reverse $c$-escape property if, for every $x \in X$, there exists $y \in\{o\} \cup \partial_{\text {ns }} X$ and $\gamma \in \Gamma_{\tau}(x, y)$ such that $\delta_{\infty}(\gamma(t)) \geq c\left(t+\delta_{\infty}(x)\right)$, for all $0 \leq t<T=\operatorname{len}_{\tau}(\gamma)$. If we can always take $y=o$ in this condition, we say that $(X, \tau, o)$ has the strong reverse c-escape property. Since not all spherical data arise via sphericalization, this generalizes the previous definition of a strong reverse escape property.

Example 3.5. Suppose that $\Omega$ is an unbounded domain in $\mathbb{R}^{n}$ that includes the origin $o$, let $\tau$ be the spherical metric (or the inner spherical metric if $\Omega$ is of bounded inner spherical diameter), and let $\partial_{\infty} \Omega=\{\infty\}$. Given $x \in \Omega$, the Euclidean line segment from $x$ to $o$ provides us with a path $\gamma_{1}$, parametrized by spherical arclength, which satisfies the estimate $\delta_{\infty}\left(\gamma_{1}(t)\right) \geq t+\delta_{\infty}(x)$. We pick $\gamma$ to be an initial segment of $\gamma_{1}$ that ends the first time that $\gamma_{1}$ hits $\partial \Omega \cup\{o\}$. Thus $(\Omega, \tau, o)$ always has a reverse 1-escape property. However, $(\Omega, \tau, o)$ may fail to have a strong reverse escape property; one example of this type, with bounded inner spherical diameter, is the domain in Example 2.32.

Example 3.6. Consider $X \subset \mathbb{R}^{2}$, where $X$ consists of the horizontal line segment $\left(x_{1}, 0\right), 0<x_{1} \leq 1$, together with vertical line segments of the form $\left(a_{n}, x_{2}\right)$, $0 \leq x_{2} \leq b_{n}, n \in \mathbb{N}$, where $a_{1}<1,\left(a_{n}\right)$ is a strictly decreasing sequence of positive numbers tending to zero, and $\left(b_{n}\right)$ is a bounded sequence of positive numbers. We equip $X$ with the inner Euclidean metric, which we denote by $\tau$. Then $\partial X_{\tau}$ contains only the single point $(0,0)$ and we define $\partial_{\infty} X=\partial X_{\tau}$. We also write $o=(1,0) \in X$. It is clear that $(X, \tau, o)$ has a (strong) reverse escape property if and only if $\left(b_{n} / a_{n}\right)$ is bounded. In particular, if we choose $a_{n}=1 /(n+1)$ ! and $b_{n}=1 / n$ !, then $(X, \tau, o)$ fails to have a reverse escape property despite the fact that it is in other ways a rather nice metric space, being for instance a compact Ahlfors regular tree.

Example 3.7. Let the data $\left(X, \tau, \partial_{\infty} X\right)$ be as in the previous example. Given $T \subset \mathbb{N}$, define $A_{T}:=\left\{\left(a_{n}, b_{n}\right) \mid n \in T\right\} \subset X, X_{T}=X \backslash A_{T}, \partial_{\mathrm{ns}} X_{T}=\partial_{\mathrm{ns}} X \cup A_{T}$, and denote by $\tau$ the subspace metric inherited by $X_{T}$. Then $\left(X_{T}, \tau, \partial_{T} X\right)$ are spherical data, and $\left(X_{T}, \tau, o\right)$ has a reverse escape property if and only if $b_{n} / a_{n}$ is uniformly bounded for $n \in \mathbb{N} \backslash T$. In the extreme case $T=\mathbb{N},\left(X_{\mathbb{N}}, \tau, o\right)$ always has a reverse 1-escape property. A strong reverse escape property is more restrictive: It always requires the full sequence $\left(b_{n} / a_{n}\right)$ to be bounded.

We pause to introduce some notation and estimates that will be used throughout the remainder of this section. Suppose $f$ is a $\left(C, d_{0}\right)$-flattening function. Given $x \in X$, let $B_{x}^{\prime}=B_{\tau}\left(x, r_{x}^{\prime}\right)$, where $r_{x}^{\prime}:=\delta_{\infty}(x) / 2$. Let

$$
L_{j}=\left\{x \in X \mid 2^{-j} d_{0}<\delta_{\infty}(x) \leq 2^{-j+1} d_{0}\right\}, \quad j \in \mathbb{N},
$$

and let $L_{-1}$ be the empty set. We also define fatter layers $L_{i, j}=\bigcup_{m=i}^{j} L_{m}$ for all $-1 \leq i \leq j \leq \infty$. We denote the $\tau$-closure of any of these sets by means of a bar: $\overline{B_{x}^{\prime}}, \bar{L}_{j}$, etc. Note that $B_{x}^{\prime} \subset L_{j-1, j+1}$ for all $x \in L_{j}$. 
It follows from (F1) that $f\left(\delta_{\infty}(y)\right) / f\left(\delta_{\infty}(x)\right) \in[1 / C, C]$ for all $y \in \overline{B_{x}^{\prime}}$, and that $f\left(\delta_{\infty}(y)\right) / f\left(\delta_{\infty}(z)\right) \in[1 / C, C]$ for all $y, z \in \bar{L}_{j}$. Thus if $\gamma$ is a path in $X$, then

$$
\begin{aligned}
C^{-1} & \leq \frac{\operatorname{len}_{l}(\gamma)}{\operatorname{len}_{\tau}(\gamma) f\left(\delta_{\infty}(x)\right)} \leq C, \quad \gamma \subset \overline{B_{x}^{\prime}} \\
C^{i-j-1} & \leq \frac{\operatorname{len}_{l}(\gamma)}{\operatorname{len}_{\tau}(\gamma) f\left(\delta_{\infty}(x)\right)} \leq C^{j+1-i}, \quad \gamma \subset \bar{L}_{i, j}, x \in \bar{L}_{i, j}, \quad 0 \leq i \leq j .
\end{aligned}
$$

If $\gamma$ is a path that passes through $x$ of $\tau$-length at least $r_{x}^{\prime}$, then it has a piece of $\tau$-length at least $r_{x}^{\prime}$ inside $B_{x}^{\prime}$, and so (3.8) implies that

$$
\operatorname{len}_{l}(\gamma) \geq \delta_{\infty}(x) f\left(\delta_{\infty}(x)\right) / 2 C
$$

In particular, this estimate holds for any path through $x$ that is not contained in $B_{x}^{\prime}$, and for any path through $x \in \bar{L}_{i}$ that is not contained in $L_{i-1, i+1}$.

We now state and prove the main result of this section which concerns the relationship between quasihyperbolic metrics associated with $\tau$ and $l$. We do not want to restrict $(X, l)$ to be incomplete, so we use $k_{l, C}$ for some $C<\infty$ as the quasihyperbolic metric associated with $l$. By contrast, only incomplete spaces $(X, \tau)$ are of interest, so we use $k_{\tau}$ as the quasihyperbolic metric associated with $\tau$. Note that

$$
\begin{aligned}
k_{\tau}(x, y) & =\inf _{\gamma \in \Gamma(x, y ; \tau)} \int_{\gamma} \frac{d \sigma(z)}{\delta_{\tau}(z)} \\
k_{l, C}(x, y) & =\inf _{\gamma \in \Gamma(x, y ; l)} \int_{\gamma} \frac{d l(z)}{\delta_{l, C}(z)}=\inf _{\gamma \in \Gamma(x, y ; l)} \int_{\gamma} \frac{f\left(\delta_{\infty}(z)\right) d \sigma(z)}{\delta_{l, C}(z)},
\end{aligned}
$$

where $d l$ and $d \sigma$ are the length elements with respect to $l$ and $\tau$, respectively. As before, $\delta_{l, C}(z)$ is defined relative to some fixed basepoint $o \in X$, and we write $|x|=l(x, o)$.

Theorem 3.11. Let $\left(X, \tau, \partial_{\infty} X\right)$ be $d_{0}$-spherical data, where $(X, \tau)$ is locally complete, let $l:=\mathfrak{L}\left(\tau, \partial_{\infty} X, f\right)$ for some $\left(C_{f}, d_{0}\right)$-flattening function $f$, let $0<C_{l}<\infty$, and define $h(t):=t f(t) / C_{l}$.

(a) $k_{l, C_{l}} \leq C^{\prime} k_{\tau}$, where $C^{\prime}$ depends only on $C_{f}$ and $h\left(\delta_{\infty}(o)\right)$.

(b) If $(X, \tau, o)$ has a reverse c-escape property, then $k_{l, C_{l}} \geq c^{\prime} k_{\tau}$ for some $c^{\prime}>0$ dependent only on $c, C_{f}$, and $h\left(d_{0}\right)$.

(c) Conversely if $k_{l, C_{l}} \geq c^{\prime} k_{\tau}$ for some $c^{\prime}>0$, then $(X, \tau, o)$ has a reverse c-escape property for some $c=c\left(c^{\prime}, C_{f}\right)$.

Proof. Since $k_{\tau}$ is the conformal deformation of $\tau$ with density function $1 / \delta_{\tau}(x)$, and $k_{l, C_{l}}$ is the conformal deformation of $\tau$ with density function $f\left(\delta_{\infty}(x)\right) / \delta_{l, C_{l}}(x)$, inequalities between $k_{l, C_{l}}$ and $k_{\tau}$ are equivalent with the corresponding reverse inequalities between $\delta_{l, C_{l}}(x)$ and $f\left(\delta_{\infty}(x)\right) \delta_{\tau}(x)$. Note also that if we replace $f$ by $s f$ and $C_{l}$ by $s C_{l}$ for any number $s>0$, then both the hypotheses and conclusions remain unchanged. This allows us to assume without loss of generality a normalization $t_{0} f\left(t_{0}\right)=1$, where $t_{0} \in\left(0, d_{0}\right]$ is any value that suits our purposes; dependence on $h\left(t_{0}\right)$ is then equivalent to dependence on $C_{l}$. 
Part (a) is equivalent to the inequality $f\left(\delta_{\infty}(x)\right) \delta_{\tau}(x) \lesssim \delta_{l, C_{l}}(x)$. We assume without loss of generality that $\delta_{\infty}(o) f\left(\delta_{\infty}(o)\right)=1$. We first wish to prove that $f\left(\delta_{\infty}(x)\right) \delta_{\tau}(x) \lesssim C_{l}+|x|$. If $\delta_{\infty}(x) \leq \delta_{\infty}(o) / 2$, then using (F1) we see that

$$
f\left(\delta_{\infty}(x)\right) \delta_{\tau}(x) \leq f\left(\delta_{\infty}(x)\right) \delta_{\infty}(x) \leq C_{f} \int_{\delta_{\infty}(x)}^{2 \delta_{\infty}(x)} f(t) d t \leq C_{f}|x| .
$$

Note that the last inequality follows by similar reasoning to that in Remark 2.8. If instead $\delta_{\infty}(x)>\delta_{\infty}(o) / 2$, then (F1), (F3), and our normalization ensure that $f\left(\delta_{\infty}(x)\right) \delta_{\tau}(x) \lesssim 1 \lesssim C_{l}$.

It remains to prove that $f\left(\delta_{\infty}(x)\right) \delta_{\tau}(x) \lesssim \delta_{l}(x)$; in particular, we may assume that $X_{l}$ is incomplete. By (3.8), a path $\gamma \in \Gamma_{\tau}(x, y), y \in \partial_{\mathrm{ns}} X=\partial X_{l}$, has l-length comparable to $\operatorname{len}_{\tau}(\gamma) f\left(\delta_{\infty}(x)\right)$ if $\gamma \subset \overline{B_{x}^{\prime}} \cup\{y\}$; this gives the desired inequality if such a path $\gamma$ has $l$-length less than $2 \delta_{l}(x)$. Alternatively if no such path exists, then we may apply (3.10) to every path $\gamma$ from $x$ to $\partial X_{l}$ to get that $\operatorname{len}_{l}(\gamma) \gtrsim f\left(\delta_{\infty}(x)\right) \operatorname{len}_{\tau}(\gamma)$. Taking a minimum over all such connecting paths $\gamma$, we deduce $(\mathrm{a})$.

The conclusion of (b) is equivalent to the inequality $\delta_{l, C_{l}}(x) \lesssim f\left(\delta_{\infty}(x)\right) \delta_{\tau}(x)$. We assume without loss of generality that $d_{0} f\left(d_{0}\right)=1$. The reverse escape property provides us with a path $\gamma \in \Gamma_{\tau}(x, y)$ such that $y \in\{o\} \cup \partial_{\mathrm{ns}} X$ and $\delta_{\infty}(\gamma(t)) \geq$ $c_{0}\left(t+\delta_{\infty}(x)\right), 0 \leq t<T=\operatorname{len}_{\tau}(\gamma) \leq d_{0}$. By this last inequality, a change of variables, (F3), and (F2), we see that

$$
\begin{aligned}
\operatorname{len}_{l}(\gamma)=\int_{0}^{T} f\left(\delta_{\infty}(\gamma(t))\right) d t & \lesssim \int_{0}^{T} f\left(c_{0}\left(t+\delta_{\infty}(x)\right)\right) d t \\
& \leq \int_{c_{0} \delta_{\infty}(x)}^{d_{0}} f(s) d s \\
& \lesssim f\left(\delta_{\infty}(x)\right) \delta_{\infty}(x) .
\end{aligned}
$$

Assuming that $\delta_{\tau}(x) \geq \delta_{\infty}(x) / 2$, it follows that $|x| \wedge \delta_{l}(x) \lesssim f\left(\delta_{\infty}(x)\right) \delta_{\tau}(x)$. The desired inequality now follows since also

$$
C_{l} \lesssim 1=d_{0} f\left(d_{0}\right) \lesssim t f(t), \quad 0<t \leq d_{0}
$$

Suppose instead that $\delta_{\tau}(x)<\delta_{\infty}(x) / 2$ and so we can find $\gamma \in \Gamma_{\tau}(x, y), y \in \partial_{\mathrm{ns}} X$, such that $\operatorname{len}_{\tau}(\gamma)<\left(2 \delta_{\tau}(x)\right) \wedge\left(\delta_{\infty}(x) / 2\right)$. Then $\gamma \subset B_{x}^{\prime} \cup\{y\}$ and by (3.8), we have $\delta_{l}(x) \leq \operatorname{len}_{l}(\gamma) \lesssim f\left(\delta_{\infty}(x)\right) \delta_{\tau}(x)$. This concludes the proof of (b).

For (c), we need to show that the inequality $\delta_{l, C_{l}}(x) \leq f\left(\delta_{\infty}(x)\right) \delta_{\tau}(x) / c^{\prime}$, $x \in X$, implies a reverse escape property. Let $x \in X$ be fixed but arbitrary. There exists a point $w_{0} \in\{o\} \cup \partial_{\mathrm{ns}} X$, and a path $\lambda_{0}:\left[0, T_{0}\right) \rightarrow X$, parametrized by $l$-arclength, such that $\lambda_{0}(0)=x, \lambda_{0}\left(T_{0}-; l\right)=w_{0}$, and $T_{0} \leq 2 f\left(\delta_{\infty}(x)\right) \delta_{\tau}(x) / c^{\prime}$. Let $\nu_{0}:\left[0, S_{0}\right) \rightarrow X$ be the reparametrization of $\lambda_{0}$ by $\tau$-arclength.

Using Lemma 3.3 and the fact that $\delta_{\infty}(\cdot) \geq \delta_{\tau}(\cdot)$, we see that $\delta_{\infty}\left(\lambda_{0}(t)\right) \gtrsim \delta_{\infty}(x)$. Next, using the bound $T_{0} \leq 2 f\left(\delta_{\infty}(x)\right) \delta_{\tau}(x) / c^{\prime}$ and the comparability of all values of $f(s)$ for $s \approx \delta_{\infty}(x)$, we see that there must exist a positive number $s_{1} \lesssim \delta_{\infty}(x)$ such that either $s_{1}=S_{0}$, or $s_{1}<S_{0}$ and $\delta_{\infty}\left(z_{1}\right)>2 \delta_{\infty}(x)$, where $z_{1}=\nu\left(s_{1}\right)$. In the first case, we have reached a stopping point, while in the second case we iterate. As in the proof of Theorem 2.28, we get the desired path by joining together the segments $\gamma_{j}$ obtained by this process; of course this time the process must stop after finitely many steps because $\delta_{\infty}(\cdot)$ is bounded. 
3.13. Flattened spaces have a strong escape property. We saw in Theorem 2.31 that if we sphericalize a length metric $l$, we get a metric $\tau$ satisfying a strong reverse escape property. We now prove the dual of this result: If we flatten a length metric $\tau$, we get a metric $l$ satisfying a strong escape property.

Theorem 3.14. Let $\left(X, \tau, \partial_{\infty} X\right)$ be $d_{0}$-spherical data, where $\tau$ is a local length metric, and let $l:=\mathfrak{L}\left(\tau, \partial_{\infty} X, f\right)$ for some $\left(C, d_{0}\right)$-flattening function $f$. Given $o \in X$, there exist $0<c<1$, and $0<\rho<\infty$ such that:

(a) $(X, l, o)$ has a $(c, \rho)$-escape property, and

(b) if $\tau$ is a length metric, then $(X, l, o)$ has a strong $(c, \rho)$-escape property.

Moreover, we can take $c$ to depend only on $C$, and $\rho$ to depend only on $C$ and $\delta_{\infty}(o) f\left(\delta_{\infty}(o)\right)$.

Proof. We first prove (b). Let us fix $o \in X$. Given $x \in X$, with $|x|$ sufficiently large, we will construct a strong escape path $\gamma$ by concatenating a sequence of path segments. First, write $x_{0}=x, S=\delta_{\infty}(x)$, and choose a path $\lambda_{0}:\left[0, S_{0}\right) \rightarrow X$, parametrized by $\tau$-arclength, with $\lambda_{0}(0)=x_{0}, \lambda_{0}\left(S_{0}-; \tau\right)=y_{0} \in \partial_{\infty} X$, and $S_{0}<2 S$. Let $x_{1}=\lambda_{0}\left(t_{1}\right)$, where $t_{1} \in\left(0, S_{0}\right)$ is such that $\delta_{\infty}\left(x_{1}\right)=S / 2$ and $\delta_{\infty}\left(\lambda_{0}(t)\right)>S / 2$ for all $0<t<t_{1}$. Let $\gamma_{1}=\left.\lambda_{0}\right|_{\left[0, t_{1}\right]}$. Inductively, given $x_{j} \in X$ with $\delta_{\infty}\left(x_{j}\right)=2^{-j} S$, we let $\lambda_{j}:\left[0, S_{j}\right) \rightarrow X$ be parametrized by $\tau$-arclength with $\lambda_{j}(0)=x_{j}, \lambda_{j}\left(S_{j}-; \tau\right)=y_{j} \in \partial_{\infty} X$, and $S_{j}<2 \delta_{\infty}\left(x_{j}\right)$. As for $j=0$, we find $x_{j+1}=\lambda_{j}\left(t_{j+1}\right)$ such that $\delta_{\infty}\left(x_{j+1}\right)=\delta_{\infty}\left(x_{j}\right) / 2$ but $\delta_{\infty}\left(\lambda_{j}(t)\right)>\delta_{\infty}\left(x_{j}\right) / 2$ for all $0<t<t_{j+1}$, and we let $\gamma_{j+1}=\left.\lambda_{j}\right|_{\left[0, t_{j+1}\right]}$. Note that $\operatorname{len}_{\tau}\left(\gamma_{j}\right)<2^{2-j} S$. Joining together the paths $\gamma_{j}, j \in \mathbb{N}$, and parametrizing the resulting curve by $l$-arclength, we get a path $\gamma:[0, \infty) \rightarrow X$ with $\gamma(0)=x$ and of infinite $l$-length (since it connects $x$ to $\partial_{\infty} X$ ). It is convenient to denote by $\gamma^{j}$ the initial segment of $\gamma$ obtained by gluing together $\gamma_{i}, i \leq j$.

By construction, $\delta_{\infty}(z) / 2^{1-j} S \in[1 / 2,2]$ for $z \in \gamma_{j}$, and so $f\left(\delta_{\infty}(z)\right) / f\left(2^{1-j} S\right) \in$ $[1 / C, C]$. Since the $\tau$-length of $\gamma_{j}$ is at most $2^{2-j} S$, we get that

$$
\operatorname{len}_{l}\left(\gamma_{j}\right) \leq C 2^{2-j} S f\left(2^{1-j} S\right) \leq 4 C^{2} \int_{2^{-j} S}^{2^{1-j} S} f(s) d s
$$

where the second inequality follows from (F1). If $z=\gamma(t) \in \gamma_{j}$, it follows from the last inequality and (F2) that

$$
t \leq \operatorname{len}_{l}\left(\gamma^{j}\right) \leq 4 C^{2} \int_{2^{-j} S}^{S} f(s) d s \leq C^{3} 2^{2-j} S f\left(2^{-j} S\right) .
$$

Our goal is to obtain a lower estimate for $|z|=\gamma(t)$ in terms of $t$. Suppose first that $z \in \gamma_{j}$, where $2^{3-j} S \leq \delta_{\infty}(o)$; in particular $\delta_{\infty}(z)<\delta_{\infty}(o)$. Given $\nu \in \Gamma_{\tau}(z, o)$, we have

$$
\operatorname{len}_{l}(\nu)=\int_{0}^{\operatorname{len} \tau(\nu)} f\left(\delta_{\infty}(\nu(t))\right) d t
$$

Applying Lemma 2.5 with $\alpha=\delta_{\infty} \circ \nu$ to this last equation and taking an infimum over all such $\nu$, we get

$$
|z| \geq \int_{\delta_{\infty}(z)}^{\delta_{\infty}(o)} f(t) d t .
$$


Since $z \in \gamma_{j}$ and $\delta_{\infty}(o) \geq 2^{3-j} S$, it follows from (F1) and (F2) that

$$
|z| \geq \int_{2^{2-j} S}^{\delta_{\infty}(o)} f(t) d t \geq \int_{2^{2-j} S}^{2^{3-j} S} f(t) d t \geq C^{-3} 2^{2-j} S f\left(2^{-j} S\right) .
$$

Putting together (3.17) and (3.15), we see that $|\gamma(t)| \geq t / C^{6}$. This last inequality readily implies that $|\gamma(t)| \geq c(t+|x|)$, where $c=1 / 3 C^{6}$; simply consider separately the possibilities $t \geq|x| / 2$ and $t<|x| / 2$, and use the triangle inequality in the latter case. Thus we have proved the defining inequality for a strong escape path on the final segment of $\gamma$ consisting of $\gamma_{j}$ for all $j \in \mathbb{N}$ such that $2^{-j} S \leq \delta_{\infty}(o) / 8$. In particular, the full path $\gamma$ is a strong escape path for $x$ if $\delta_{\infty}(x)<\delta_{\infty}(o) / 4$.

Suppose instead that $z \in \gamma_{j}$, where $2^{3-j} S>\delta_{\infty}(o)$. According to (3.15), $\operatorname{len}_{l}\left(\gamma^{j}\right) \leq C^{3} 2^{-j+2} S f\left(2^{-j} S\right)$. Since $2^{-j} S \gtrsim \delta_{\infty}(o)$, it follows from this last inequality, (F1), and (F3) that $\operatorname{len}_{l}\left(\gamma^{j}\right) \leq C^{\prime}$ for some $C^{\prime} \lesssim 1$. It follows that if $|x| \geq \rho:=2 C^{\prime}$, then $|z| \geq(t+|x|) / 3$.

The proof of (a) is similar, but let us comment briefly on the necessary alterations in the construction of the escape path $\gamma$. First, $S=\delta_{\tau}(x)$ and $\lambda_{0}:\left[0, S_{0}\right) \rightarrow X$ is parametrized by $\tau$-arclength and satisfies $\lambda_{0}(0)=x_{0}, \lambda_{0}\left(S_{0}-; \tau\right)=y_{0} \in \partial_{\tau} X$, and $S_{0}<2 S$. Then $x_{1}=\lambda_{0}\left(t_{1}\right)$, where $t_{1}$ is the least value of $t$ in $\left(0, S_{0}\right)$ for which $\delta_{\infty}\left(\lambda_{0}(t)\right)=S / 2$, or $t_{1}=S_{0}$ if no such number exists. In the latter case the construction ends. If the construction has not yet ended, we are inductively given $x_{j} \in X$ with $\delta_{\tau}\left(x_{j}\right)=2^{-j} S$. We let $\lambda_{j}:\left[0, S_{j}\right) \rightarrow X$ be parametrized by $\tau$-arclength with $\lambda_{j}(0)=x_{j}, \lambda_{j}\left(S_{j}-; \tau\right)=y_{j} \in \partial_{\tau} X$, and $S_{j}<2 \delta_{\infty}\left(x_{j}\right)$. As for $j=0$, we let $x_{j+1}=\lambda_{j}\left(t_{j+1}\right)$, where $t_{j+1}$ is the least value of $t \in\left(0, S_{j}\right)$ such that $\delta_{\infty}\left(\lambda_{j}(t)\right)=\delta_{\infty}\left(x_{j}\right) / 2$, or $t_{j+1}=S_{j}$ if no such number exists. In the latter case, the construction ends.

We cannot conclude in Theorem 3.14(a) that $(X, l, o)$ has a strong $c$-escape property. For instance, if $X \subset \mathbb{R}^{2}$ is given by the curve complement

$$
\left\{(s, t) \mid s, t \in \mathbb{R}, s \geq 1, t \neq s^{2} \sin ^{2} s\right\} \subset \mathbb{R}^{2},
$$

with the spherical metric attached, $\partial_{\infty} X=\{\infty\}$, and we use the standard flattening function $f$, then $l=\mathfrak{L}\left(\tau, \partial_{\infty} X, f\right)$ is the inner Euclidean metric on $X$, and $(X, l, o)$ fails to have any strong $c$-escape property, as we can see by taking $x=$ $\left((2 j+1) \pi / 2, j^{2}\right)$ for sufficiently large $j=j_{c} \in \mathbb{N}$.

\section{Are sphericalization and FLATtening inVerse processes?}

In this section, we want to discuss whether or not we get a bilipschitz equivalent metric back when we sphericalize a flattened metric, or flatten a sphericalized metric. Since both sphericalized and flattened metrics are length metrics, we always assume we are dealing with length metrics. Thus we shall use sphericalization and flattening to go back and forth between a pair of length metrics $l$ and $\sigma$.

Let us introduce some notation and terminology to aid us in our discussion. Throughout this section, we write $\mathcal{A}=\mathcal{U} \cup \mathcal{B}$, where $\mathcal{U}$ is the class of all unbounded pointed length spaces $(X, l, o)$ for which $o$ has a strong escape property, and $\mathcal{B}$ is the class of all bounded incomplete pointed length spaces $(X, \sigma, o)$ with associated spherical data. Fixing a sphericalization function $g$ and a flattening function $f$, we have seen that sphericalization associates an element of $\mathcal{B}$ with each element of $\mathcal{U}$, and that flattening associates an element of $\mathcal{U}$ with each element of $\mathcal{B}$. In each case let us call the associated space the $s f$-dual of the original space. More formally, we 
should talk of original and dual data since flattening requires spherical data to be specified, but we prefer to refer to the spherical data and the functions $f, g$ only when needed. We also call a space a bsf-dual if it is bilipschitz equivalent to the sf-dual of some space.

We say that a space in $\mathcal{A}$ is sf-reflexive if it is bilipschitz equivalent to its double sf-dual. For instance Euclidean space and the Riemann sphere (with north pole omitted) are sf-reflexive with respect to the standard sphericalization and flattening functions. It is clear that sf-reflexivity requires that the functions $f, g$ satisfy some sort of duality relation; the desired condition, which we call quasiduality will be defined in $§ 4.2$. A necessary condition for a space to be sf-reflexive is that it is a bsf-dual. We now state the main theorem in this last section which says that being a bsf-dual is also a sufficient condition for a space to be sf-reflexive.

Theorem 4.1. Suppose $X$ is a space in the class $\mathcal{A}$ and sf-duality is defined via quasidual flattening and sphericalizing functions $f, g$. Then the following are equivalent:

(a) $X$ is sf-reflexive.

(b) $X$ is a bsf-dual.

(c) $(X, l, o)$ has a strong escape property $($ if $(X, l, o)$ is in $\mathcal{U})$ or $(X, \sigma, o)$ has a strong reverse escape property (if $(X, \sigma, o)$ is in $\mathcal{B})$.

This result says, in particular, that the sf-dual of every space in $\mathcal{A}$ is sf-reflexive. However not all spaces in $\mathcal{A}$ are sf-reflexive. Consider for example the spaces $\left(X_{T}, l, o\right)$ in Example 2.17 and the spaces $\left(X_{T}, \sigma, o\right)$ in Example 3.7. According to Theorem 4.1, these spaces are sf-reflexive if and only if the full sequence $\left(b_{n} / a_{n}\right)_{n=1}^{\infty}$ is bounded. For counterexamples in $\overline{\mathbb{R}^{n}}$, see Examples 2.14 and 2.32 .

4.2. Quasiduality. For a space to be sf-reflexive, with respect to a particular choice of sphericalization and flattening functions $g$ and $f$, requires something of the geometry of the space as indicated in Theorem 4.1, but it also requires that $g$ and $f$ are in some sense well matched. For instance, we will see that the "standard" sphericalization and flattening functions $g(t)=2 /\left(1+t^{2}\right), 0 \leq t<\infty$, and $f(t)=$ $\csc ^{2}(t / 2) / 2,0<t \leq d_{0}:=\pi$, are quasidual. Since $f(t)$ is comparable with $t^{-2}$, this is essentially a special case of the fact that for $p, q>1, d_{0}>0, g_{p}(t):=1 /\left(1+t^{p}\right)$ is comparable with $f_{q}(t):=t^{-q}, 0<t \leq d_{0}$, if and only if $q=p /(p-1)$. Before we formally define the notion of quasiduality, let us consider Euclidean space and the Riemann sphere as motivational examples.

Let $X=\mathbb{R}^{n}, n>1$, with the Euclidean metric $l$ attached, and let $o$ be the usual origin. If $\sigma=\mathfrak{S}(l, o, g)$ for some sphericalization function $g$, then the resulting space $(X, \sigma)$ is topologically a sphere minus a single point $w$ at infinity (the only element of the spherical boundary). Moreover rays from $o$ are geodesic both for $l$ and $\sigma$, and $\delta_{\infty}(x)=\sigma(x, w)=\int_{|x|}^{\infty} g(s) d s$. If we now define $l^{\prime}=\mathfrak{L}(\sigma,\{w\}, f)$ for some flattening function $f$ whose $d_{0}$ parameter is at least as large as $\int_{0}^{\infty} g(s) d s$, then

$$
d l^{\prime}(x)=f\left(\delta_{\infty}(x)\right) d \sigma(x)=f\left(\int_{|x|}^{\infty} g(s) d s\right) \cdot g(|x|) d l(x) .
$$


Thus $l^{\prime}$ is bilipschitz equivalent to $l$ if and only if there is some constant $C \geq 1$ such that

$$
\frac{1}{C} \leq f\left(\int_{t}^{\infty} g(s) d s\right) \cdot g(t) \leq C, \quad 0 \leq t<\infty .
$$

In the opposite direction, let us begin with $X=\overline{\mathbb{R}^{n}} \backslash\{\infty\}, n>1$, the Riemann sphere with the north pole removed, and let $d_{0}=\pi$. Flattening and then sphericalizing using some flattening and sphericalization functions $f:\left[0, d_{0}\right] \rightarrow(0, \infty)$ and $g$, respectively, we get a new metric $\sigma^{\prime}$ which is comparable to $\sigma$ if and only if there is some constant $C \geq 1$ such that

$$
\frac{1}{C} \leq g\left(\int_{t}^{d_{0}} f(s) d s\right) \cdot f(t) \leq C, \quad 0<t \leq d_{0} .
$$

We leave the straightforward verification of the above statements to the reader.

We could use (4.3) and (4.4) as our definition of quasiduality, but we would like to replace them by quantitatively equivalent conditions that are simpler to verify and to use. Note that (2.3) tells us that $\int_{t}^{\infty} g(s) d s$ is comparable with $t g(t)$ when $t \geq 1$, so using also (F1), we see that (4.3) can be recast (at least for $t \geq 1$ ) as

$$
f(t g(t)) \operatorname{tg}(t) \approx t, \quad 1 \leq t<\infty,
$$

as long as $f(t g(t))$ is always defined. Similarly (4.4) can be recast as

$$
g(t f(t)) t f(t) \approx t, \quad 0<t \leq d_{0} / 2 .
$$

Our official definition of quasiduality, which we now give, is similar to this last pair of conditions, but modified to ensure that all function values are defined.

Definition 4.7. Suppose that $C \geq 1, d_{0}>0$, and that $f:\left(0, d_{0}\right] \rightarrow(0, \infty)$ and $g:[0, \infty) \rightarrow(0, \infty)$ are continuous. Let $F, G$ be defined by the equations

$$
\begin{aligned}
& F(t)= \begin{cases}t f(t), & 0<t<d_{0}, \\
d_{0}^{2} f\left(d_{0}\right) / t, & d_{0} \leq t,\end{cases} \\
& G(t)= \begin{cases}g(1) / t, & 0<t<1, \\
t g(t), & 1 \leq t .\end{cases}
\end{aligned}
$$

We say that $f$ is a left $C$-quasidual of $g$, or that $g$ is a right $C$-quasidual of $f$ if $F(G(t)) \in[t / C, C t]$ for all $0<t<\infty$. We say that $f$ is a right $C$-quasidual of $g$, or that $g$ is a left $C$-quasidual of $f$, if $G(F(t)) \in[t / C, C t]$ for all $0<t<\infty$. We say that $f$ and $g$ are $C$-quasidual if $f$ is a left and a right $C$-quasidual of $g$.

It is easy to see that quasiduality remains true (with a quantitatively controlled change in the parameter $C$ ) if the sphericalization and flattening functions $g$ and $f$ are replaced by comparable functions, and $d_{0}$ is replaced by a comparable positive number, for instance if the standard choices $g(t)=2 /\left(1+t^{2}\right), 0 \leq t<\infty$, and $f(t)=\csc ^{2}(t / 2) / 2,0<t \leq \pi$, are replaced by $\tilde{g}(t)=1 \wedge t^{-2}, 0 \leq t<\infty$, and $\tilde{f}(t)=t^{-2}, 0<t \leq 1$. Verifying quasiduality for $\tilde{f}$ and $\tilde{g}$ is trivial. More generally, if $p, q>1, d_{0}>0$, then $g(t)=1 /\left(1+t^{p}\right)$ is comparable with $t^{-p}$ for $1 \leq t<\infty$, and so it is clear that $g$ is quasidual to $f(t)=t^{-q}, 0<t \leq d_{0}$, if and only if $q=p /(p-1)$.

The statement of Theorem 4.1 leads naturally to the following question: Do all sphericalizing and flattening functions have quasidual flattening and sphericalizing 
functions, respectively? We will see that the answer is affirmative. In fact, as we will see later, it is rather easy to prove that sphericalizing and flattening functions have one-sided quasiduals but this then leads to the following pair of questions. Are one-sided quasiduals of sphericalizing and flattening functions automatically two-sided quasiduals? Are quasiduals of sphericalizing and flattening functions automatically flattening and sphericalizing functions, respectively? We next state four lemmas which essentially give affirmative answers to these two questions.

Lemma 4.10. Suppose that $f:\left(0, d_{0}\right] \rightarrow(0, \infty)$ is a $C_{f}$-flattening function, and that $g:[0, \infty) \rightarrow(0, \infty)$ is a left or right $C_{g}$-quasidual of $f$. Then $g$ is a $C$-quasidual of $f$ for some $C=C\left(C_{f}, C_{g}\right)$.

Lemma 4.11. Suppose that $g$ is a $C_{g}$-sphericalizing function and that $f:\left(0, d_{0}\right] \rightarrow$ $(0, \infty)$ is a left or right $C_{f}$-quasidual of $g$. Then $f$ is a $C$-quasidual of $g$, for some $C=C\left(C_{f}, C_{g}\right)$.

Lemma 4.12. Suppose that $f:\left(0, d_{0}\right] \rightarrow(0, \infty)$ is a $C_{f}$-quasidual of a $C_{g^{-}}$ sphericalizing function $g$. Then $f$ is a $\left(C, d_{0}\right)$-flattening function for some $C=$ $C\left(C_{f}, C_{g}\right)$.

Lemma 4.13. Suppose that $g$ is a $C_{g}$-quasidual of a $\left(C_{f}, d_{0}\right)$-flattening function $f$, and that $g(r) / g(s) \in\left[1 / C_{g}, C_{g}\right]$ for all $0 \leq r, s \leq 1$. Then $g$ is a $C$-sphericalizing function for some $C=C\left(C_{f}, C_{g}\right)$.

Note that comparability of the values of $g$ on $[0,1]$ needs to be assumed in this last lemma since quasiduality tells us nothing about these values. The proof of Lemma 4.11 is very similar to that of Lemma 4.10, and the proof of Lemma 4.13 is very similar to that of Lemma 4.12, so we omit both of them.

Before proving Lemmas 4.10 and 4.12, it is useful to rewrite the basic assumptions (F1), (F3), (S1), and (S3) as decay estimates for the functions $F$ and $G$ in (4.8) and (4.9). Suppose $f$ is a $\left(C_{f}, d_{0}\right)$-flattening function and $g$ is a $C_{g}$-sphericalizing function. Then there are positive constants $K_{f}, K_{g}, C_{f}^{\prime}, C_{g}^{\prime}, \epsilon_{f}, \epsilon_{g}$ such that

$$
\begin{array}{rlrl}
\frac{1}{C_{f}}\left(\frac{s}{r}\right)^{K_{f}} & \leq \frac{F(r)}{F(s)} \leq C_{f}^{\prime}\left(\frac{s}{r}\right)^{\epsilon_{f}}, & 0<s \leq r<\infty, \\
\frac{1}{C_{g}}\left(\frac{s}{r}\right)^{K_{g}} \leq \frac{G(r)}{G(s)} \leq C_{g}^{\prime}\left(\frac{s}{r}\right)^{\epsilon_{g}}, & 0<s \leq r<\infty .
\end{array}
$$

Indeed, we can pick $K_{f}=\log _{2} C_{f}, K_{g}=\log _{2} C_{g}$, let $C_{f}^{\prime}$ and $\epsilon_{f}$ be the constants $C^{\prime}$ and $\epsilon$ in (F3), and let $C_{g}^{\prime}$ and $\epsilon_{g}$ be the constants $C^{\prime}$ and $\epsilon$ in (G3). We leave it to the reader to verify these estimates.

Proof of Lemma 4.10. Let $F, G$ be defined by (4.8) and (4.9). Suppose that $g$ is a right quasidual of $f$, and so $F(G(t)) \approx t, t>0$. Taking $t=F(s)$, we get that $F(G(F(s))) \approx F(s)$ for all $s>0$. Using (4.14) we see that if $F(r) \approx F\left(r^{\prime}\right)$, then $r \approx r^{\prime}$, and so we deduce that $G(F(s)) \approx s$ for all $s>0$, as required.

Suppose instead that $g$ is a left quasidual of $f$, and so $G(F(t)) \approx t, t>0$. Again by (4.14), we see that if $t \approx t^{\prime}$, then $F(t) \approx F\left(t^{\prime}\right)$, and so $F(G(F(t))) \approx F(t)$. But it is clear from the definition of $F$ and (F3) that $F$ has range $(0, \infty)$, and so $F(G(s)) \approx s$ for all $s>0$. 
Proof of Lemma 4.12. Throughout this proof, we assume that $0<s \leq s^{\prime} \leq d_{0}$, $t, t^{\prime}>0, G(t)=s$, and $G\left(t^{\prime}\right)=s^{\prime}$. Since $G$ has range $(0, \infty)$, such numbers $t, t^{\prime}>0$ exist regardless of the values of $r, r^{\prime}$. Quasiduality implies the useful estimate

$$
\frac{t^{\prime} F(s)}{t F\left(s^{\prime}\right)}=\frac{t^{\prime} F(G(t))}{t F\left(G\left(t^{\prime}\right)\right)} \approx 1
$$

To prove (F1), it suffices to show that if $s, s^{\prime}$ satisfy the additional constraint $s^{\prime} \leq 2 s$, then $f(s) \approx f\left(s^{\prime}\right)$, or equivalently $F(s) \approx F\left(s^{\prime}\right)$. Using (4.15), it follows that $t \approx t^{\prime}$ whenever $s^{\prime} / s \in[1,2]$. Thus using (4.16), we see that $F(s) \approx F\left(s^{\prime}\right)$, as required.

It remains to prove (F3), or equivalently $F(s) / F\left(s^{\prime}\right) \gtrsim\left(s^{\prime} / s\right)^{\delta}$ for some $\delta>0$. This already follows from (F1) if $s \approx s^{\prime}$, so we may assume that $s^{\prime} / s$ is so large that the second inequality in (4.15) implies that $t^{\prime} \leq t$. But now by the first part of (4.15), we get $t / t^{\prime} \gtrsim\left(s^{\prime} / s\right)^{1 / K_{g}}$ and, combining this inequality with (4.16), we deduce that $F(s) / F\left(s^{\prime}\right) \gtrsim\left(s^{\prime} / s\right)^{1 / K_{g}}$.

By data dilation (by a factor $a>0$ ), we will mean replacing $f$ by $t \mapsto a f(a t), g$ by $t \mapsto g(t) / a$, and $d_{0}$ by $d_{0} / a$. Data dilation by a factor $a$ leads to the associated functions $F$ and $G$ being replaced by $t \mapsto F(a t)$ and $t \mapsto a^{-1} G(t)$, respectively. It follows that $C$-quasiduality is invariant under data dilations. Note also that the assumptions that $g$ is a $C$-sphericalizing function and that $f$ is a $\left(C, d_{0}\right)$-flattening function for some $d_{0}>0$ are invariant under data dilations. Data dilation is used later to assume without loss of generality that $d_{0}=1$, or to normalize some value of $f$ or $g$.

With the aid of the above lemmas, it is now a straightforward task to prove the following result which answers the remaining one of the three questions posed above.

Proposition 4.17. Suppose $d_{0}>0$. Given a $C_{1}$-sphericalizing function $g$, there exists a $\left(C_{2}, d_{0}\right)$-flattening function $f$ which is $C_{3}$-quasidual to $g$. If instead we are given a $\left(C_{1}, d_{0}\right)$-flattening function $f$, there exists a $C_{2}$-sphericalizing function $g$ which is $C_{3}$-quasidual to $f$. For both statements $C_{2}$ and $C_{3}$ depend only on $C_{1}$.

Proof. By data dilation, we may assume that $g(1)=1$. Suppose that $g$ is a $C_{1}$ sphericalizing function and that $G$ is defined by (4.9). Since $G$ is continuous with range $(0, \infty)$ there exists, for any given $t>0$, a least nonnegative number $s=: H(t)$ for which $G(s)=t / d_{0}$. Note that $H(t)=d_{0} / t$ for all $t \geq d_{0}$, and $H(t)>1$ for all $t<d_{0}$. Now $H$ is strictly decreasing but it might be discontinuous on $\left(0, d_{0}\right]$. To fix this, let $F(t)=H(t)$ for all $t \geq d_{0}$ and also for $t=2^{-j} d_{0}, j \in \mathbb{N}$, and then define $F(t)$ by linear interpolation for all other $t \in\left(0, d_{0}\right)$. If we define $f(t)=F(t) / t$ for $0<t \leq d_{0}$, then $f$ and $F$ are related as in (4.8). It follows from (4.15) that the values of $H$, and hence those of $f$, are mutually comparable on each of the subintervals $I_{j}=\left[2^{-j} d_{0}, 2^{-j+1} d_{0}\right], j \in \mathbb{N}$. Using (S1), it now follows that $f$ is a right quasidual of $g$. Using Lemmas 4.11 and 4.12 , we deduce that $f$ is both a quasidual of $g$ and a flattening function.

We omit the analogous proof of the second part of this result.

4.18. Proof of Theorem 4.1. We first prove that a space $(X, l, o)$ in $\mathcal{U}$ is sfreflexive with respect to a quasidual pair $f, g$ if and only if $(X, l, o)$ has a strong escape property. 
Theorem 4.19. Let $(X, l, o)$ be an incomplete pointed length space, and let $\sigma:=$ $\mathfrak{S}(l, o, g)$, where $g$ is a $C$-sphericalizing function with a $C$-quasidual $f:\left(0, d_{0}\right] \rightarrow$ $(0, \infty), d_{0}=\operatorname{dia}_{\sigma}(X)$, and $\partial_{\infty} X$ is nonempty. Also let $\partial_{\infty} X=\partial X_{\sigma} \backslash \partial X_{l}$, and $l^{\prime}=\mathfrak{L}\left(\sigma, \partial_{\infty} X, f\right)$. Then:

(a) $l^{\prime} \leq C^{\prime} l$, where $C^{\prime}=C^{\prime}(C)$.

(b) If $(X, l, o)$ has the strong $\left(c_{0}, \rho_{0}\right)$-escape property, then $l^{\prime} \geq c l$, for some $c=c\left(C, c_{0}, r_{0}\right)>0$.

(c) If $l^{\prime} \geq c l$ for some $c>0$, then $(X, l, o)$ has the strong $\left(c_{0}, \rho_{0}\right)$-escape property, for some $c_{0}, \rho_{0}$ dependent only on $c$ and $C$.

Proof. Let $F, G$ be as defined in (4.8) and (4.9). Propositions 2.6 and 2.20 ensure that $d_{0} \leq 4 C g(1)$ and that $\left(X, \sigma, \partial_{\infty} X\right)$ are $d_{0}$-spherical data. Thus it makes sense to talk of $l^{\prime}$. Note that Lemma 4.12 implies that $f$ is a $C^{\prime \prime}$-flattening function for some $C^{\prime \prime} \lesssim 1$. Since $l^{\prime}$ is the conformal deformation of $\sigma$ with density function $f\left(\delta_{\infty}(x)\right)$, and $\sigma$ is the conformal deformation of $l$ with density function $g(|x|)$, the conclusions in (a) and (b) are equivalent to the statements that $f\left(\delta_{\infty}(x)\right) g(|x|) \lesssim 1$ and $f\left(\delta_{\infty}(x)\right) g(|x|) \gtrsim 1$, respectively. Since the hypotheses and conclusions are invariant under data dilation, we may assume that $g(1)=1$. By Proposition 2.6 and (2.9), we see that $d_{0} \approx 1$, and by quasiduality, $f\left(d_{0}\right) \approx 1$.

By $(2.22)$ and $(\mathrm{S} 2), \delta_{\infty}(x) \gtrsim G(|x|)$ for $|x| \geq 1$ and $\delta_{\infty}(x) \gtrsim g(1)=1$ for $|x|<1$. Using quasiduality, we see that $f\left(\delta_{\infty}(x)\right) g(|x|) \lesssim F(G(|x|)) /|x| \approx 1$ for $|x| \geq 1$, and that $f\left(\delta_{\infty}(x)\right) g(|x|) \lesssim F(G(1)) \approx 1$ for $|x|<1$. Thus we have proven (a).

Similarly, the conclusion of (b) follows once we prove that $\delta_{\infty}(x) \lesssim 1$ for $|x| \leq \rho_{0}$ and $\delta_{\infty}(x) \lesssim G(|x|)$ for $|x| \geq \rho_{0}$. The first estimate is trivial since $\delta_{\infty}(x) \leq d_{0} \approx$ $1 \approx \rho_{0}$. If instead $|x| \geq \rho_{0}$, then we choose a strong $c_{0}$-escape path for $x$, and (2.21) tells us that $\operatorname{len}_{\sigma}(\gamma) \lesssim G(|x|)$. Since $\gamma$ has finite $\sigma$-length but infinite $l$-length, it connects $x$ to $\partial_{\infty} X$, and so $\delta_{\infty}(x) \leq \operatorname{len}_{\sigma}(\gamma) \lesssim G(|x|)$.

Finally we prove (c). Suppose $l^{\prime} \gtrsim l$ and so $f\left(\delta_{\infty}(x)\right) g(|x|) \gtrsim 1$. By quasiduality of $f$ and $g, f(G(|x|)) g(|x|) \approx 1,|x| \geq 1$, and (F3) ensures that there exists $1 \leq$ $\rho_{0} \lesssim 1$ such that $\delta_{\infty}(x) \lesssim G(|x|)$ for all $|x| \geq \rho_{0}$. Thus if $|x| \geq \rho_{0}$, we can find a path $\lambda_{0}:\left[0, T_{0}\right) \rightarrow X$, parametrized by $\sigma$-arclength, such that $\lambda_{0}(0)=x, \lambda_{0}\left(T_{0}-; \sigma\right)=$ $w_{0} \in \partial_{\infty} X$, and $T_{0} \lesssim g(|x|)|x|$. Arguing as in Theorem 2.28, we get a strong escape path. We leave the details to the reader; note that the stopping condition never occurs since all paths $\lambda_{i}$ are of infinite $l$-length.

We next tackle the reverse direction: flattening followed by sphericalization.

Theorem 4.20. Suppose that $\left(X, \sigma, \partial_{\infty} X\right)$ are $d_{0}$-spherical data and that $l:=$ $\mathfrak{L}\left(\sigma, \partial_{\infty} X, f\right)$, where $f$ is a $\left(C, d_{0}\right)$-flattening function with a $C$-quasidual $g$. Fix $o \in X$ and define $\sigma^{\prime}:=\mathfrak{S}(l, o, g)$. Then:

(a) $\sigma^{\prime} \leq C^{\prime} \sigma$, for some $C^{\prime}$ dependent only on $C, d_{0} f\left(d_{0}\right)$, and $\delta_{\infty}(o) / d_{0}$.

(b) If $(X, \sigma, o)$ satisfies a strong reverse $c_{0}$-escape property, then $\sigma^{\prime} \geq c \sigma$, for some $c=c\left(C, c_{0}, d_{0} f\left(d_{0}\right)\right)$.

(c) If $\sigma^{\prime} \geq c \sigma, c>0$, then $(X, \sigma, o)$ satisfies a strong reverse $c_{0}$-escape property, for some $c_{0}=c_{0}\left(C, c, d_{0} f\left(d_{0}\right)\right)$.

Proof. According to Lemma 4.13, $g$ is a $C^{\prime \prime}$-sphericalizing function for some $C^{\prime \prime}=$ $C^{\prime \prime}(C)$. We write $|x|=l(x, o)$ as usual, and also write $t B_{y}=B_{\sigma}\left(y, t \delta_{\infty}(y) / 4\right)$, for all $y \in X, t>0$. The combination of data dilation by a factor $a$ and dilation of $\sigma$ distances by a factor $a^{-1}$ leaves both the assumptions and the conclusions invariant, 
so we may assume that $d_{0}=1$. Thus $F(1)=f(1)$ and, because of the dependence of all conclusions on $d_{0} f\left(d_{0}\right)$, we may assume that $F(1) \approx 1$ throughout.

By quasiduality, it follows that

$$
g\left(F\left(\delta_{\infty}(x)\right)\right) f\left(\delta_{\infty}(x)\right) \approx 1 .
$$

In fact this is immediate when $F\left(\delta_{\infty}(x)\right) \geq 1$, and it holds in general because $F\left(\delta_{\infty}(x)\right) \gtrsim F(1) \approx 1$. But, as in the proof of the previous theorem, the conclusions in (a) and (b) are equivalent to the statements that $g(|x|) f\left(\delta_{\infty}(x)\right) \lesssim 1$ and $g(|x|) f\left(\delta_{\infty}(x)\right) \gtrsim 1$, respectively. In view of (4.21), (S1), and (S3), the conclusions in (a) and (b) are thus equivalent to the statements that $F\left(\delta_{\infty}(x)\right) \lesssim|x| \vee 1$ and $F\left(\delta_{\infty}(x)\right) \gtrsim|x| \vee 1$, respectively.

Since $f$ is flattening, the values $f\left(\delta_{\infty}(z)\right), z \in 2 B_{x}$, are all mutually comparable. Therefore the $l$-arclength of a path in any such ball is comparable to $f\left(\delta_{\infty}(x)\right)$ times its $\sigma$-arclength. Thus if we define $r_{x}$ to be the largest radius with the property that $B_{x} \supset B_{l}\left(x, r_{x}\right)$, and $R_{x}$ to be the smallest radius with the property that $2 B_{x} \subset B_{l}\left(x, R_{x}\right)$, then $r_{x} \approx R_{x} \approx F\left(\delta_{\infty}(x)\right)$.

Let us now prove (a). If $x \in X,|x| \geq R_{o}$, then $x \notin 2 B_{o}$. Thus $\delta_{\infty}(x) \leq$ $\sigma(x, o)+\delta_{\infty}(o) \leq 3 \sigma(x, o)$, and so $o \notin B_{x}$. Thus $|x| \geq r_{x} \approx F\left(\delta_{\infty}(x)\right)$. It remains to show that $F\left(\delta_{\infty}(x)\right) \lesssim|x| \vee 1$ when $|x|<R_{o}$. Since $R_{o} \lesssim F\left(\delta_{\infty}(o)\right) \approx 1$, this follows from the case $|x|=R_{o}$ (which we already know) and (F1), once we show that $\delta_{\infty}(x)$ is approximately constant on $\bar{B}_{l}\left(o, R_{o}\right)$. Certainly $\delta_{\infty}(x) \leq d_{0}=1 \approx \delta_{\infty}(o)$. If $\delta_{\infty}(x)<t$ for some $t$ much smaller than $\delta_{\infty}(x)$, then we can apply Lemmas 2.5 and 3.3 to deduce that

$$
|x| \geq \int_{t}^{\delta_{\infty}(o)} f(s) d s \geq K \delta_{\infty}(0) f\left(\delta_{\infty}(o)\right) \approx K,
$$

where $K \rightarrow \infty$ as $t / \delta_{\infty}(o) \rightarrow 0$. But $|x| \leq R_{o}$, so the resulting upper bound on $K$ translates into a lower bound on $t$, as required.

We now turn to (b). Let $\gamma$ be the path from $x$ to $o$ provided by the strong reverse escape property. Since $S:=\operatorname{len}_{\sigma}(\gamma) \leq \delta_{\infty}(o) / c_{0}-\delta_{\infty}(x) \leq 1 / c_{0}-\delta_{\infty}(x)$, we see by a change of variables, (F1), (F3), and (F2) that

$$
\begin{aligned}
\operatorname{len}_{l}(\gamma)=\int_{0}^{S} f\left(\delta_{\infty}(\gamma(t))\right) d t & \lesssim \int_{0}^{S} f\left(c_{0}\left(t+\delta_{\infty}(x)\right)\right) d t \\
& =\int_{c_{0} \delta_{\infty}(x)}^{1} f(s) d s \\
& \lesssim F\left(\delta_{\infty}(x)\right) .
\end{aligned}
$$

Thus $|x| \lesssim F\left(\delta_{\infty}(x)\right)$ and (b) follows.

Finally, we prove (c). The hypothesis $\sigma^{\prime} \gtrsim \sigma$ is, as we have seen, equivalent to the statement that $F\left(\delta_{\infty}(x)\right) \gtrsim|x| \vee 1$. Thus we can find a path $\lambda_{0}:\left[0, T_{0}\right] \rightarrow X$, parametrized by $l$-arclength, such that $\lambda_{0}(0)=x, \lambda_{0}\left(T_{0}\right)=o$, and $T_{0} \lesssim F\left(\delta_{\infty}(x)\right)$. Arguing as in Theorem 3.11, we get a strong reverse escape property for $(X, \sigma, o)$; the details are left to the reader.

It is now straightforward to complete the proof of Theorem 4.1. The fact that (a) implies (b) is trivial, and (b) implies (c) according to Theorems 2.31 and 3.14. Finally, (c) implies (a) according to Theorems 4.19 and 4.20. 


\section{ACKNOWLEDGMENT}

We would like to thank the referee for carefully reading the paper and for alerting us to a problem with our original definition of a local length space.

\section{REFERENCES}

[AG] K. Astala and F. W. Gehring, Quasiconformal analogues of theorems of Koebe and HardyLittlewood, Mich. Math. J. 32 (1985), 99-107. MR0777305 (86j:30029)

[AK] K. Astala and P. Koskela, Quasiconformal mappings and global integrability of the derivative, J. Anal. Math. 57 (1991), 203-220. MR1191747(94c:30026)

[BB] Z. M. Balogh and S.M. Buckley, Geometric characterizations of Gromov hyperbolicity, Invent. Math 153 (2003), 261-301. MR 1992014 (2004i:30042)

[BK] Z. M. Balogh and P. Koskela, Quasiconformality, quasisymmetry and removability in Loewner spaces, Duke Math. J. 101 (2000), 554-577. MR1740689 (2001d:30029)

[BHK] M. Bonk, J. Heinonen, and P. Koskela, Uniformizing Gromov hyperbolic spaces, Astérisque 270 (2001). MR1829896 (2003b:30024)

[BHR] M. Bonk, J. Heinonen, and S. Rohde, Doubling conformal densities, J. reine angew. Math. 541 (2001), 117-141. MR.1876287 (2002k:30036)

[BKo] M. Bonk and P. Koskela, Conformal metrics and the size of the boundary, Amer. J. Math. 124 (2002), 1247-1287. MR1939786(2003i:30068)

[BKR] M. Bonk, P. Koskela, and S. Rohde, Conformal metrics on the unit ball in Euclidean space, Proc. London Math. Soc. 77 (1998), 635-664. MR1643421 (99f:30033)

[HK] J. Heinonen and P. Koskela, Quasiconformal maps in metric spaces with controlled geometry, Acta Math. 181 (1998), 1-61. MR1654771 (99j:30025)

[He1] D. Herron, Quasiconformal deformations and volume growth, preprint.

[He2] D. Herron, Conformal deformations of uniform Loewner spaces, Math. Proc. Camb. Phil. Soc. 136 (2004), 325-360. MR2040578 (2005i:30029)

[Se1] S. Semmes, Bilipschitz mappings and strong $A_{\infty}$ weights, Ann. Acad. Sci. Fenn. Ser. A. I. Math. 18 (1993), 211-248. MR.1234732 (95g:30032)

[Se2] S. Semmes, On the nonexistence of bilipschitz parametrization and geometric problems about $A_{\infty}$ weights, Rev. Math. Iberoamericana 12 (1996), 345-360.

[Ty1] J. T. Tyson, Quasiconformality and quasisymmetry in metric measure spaces, Ann. Acad. Sci. Fenn. Math. 23 (1998), 525-548. MR1642158 (99i:30038)

[Ty2] J. T. Tyson, Metric and geometric quasiconformality in Ahlfors regular Loewner spaces, Conform. Geom. Dyn. 5 (2001), 21-73. MR1872156 (2002m:30026)

[V] J. Väisälä, Lectures on n-dimensional quasiconformal mappings, Lecture Notes in Mathematics 229, Springer-Verlag, Berlin, 1971. MR0454009 (56:12260)

Departament Mathematik, Universität Bern, Sidlerstrasse 5, 3012, Bern, Schweiz

E-mail address: zoltan@math-stat.unibe.ch

Department of Mathematics, National University of Ireland, Maynooth, Co. KilDARE, IRELAND

E-mail address: sbuckley@maths.nuim.ie 